Wisam Alame and Wojciech M. ZajączKowski (Warszawa)

\title{
LARGE TIME REGULAR SOLUTIONS TO THE MHD EQUATIONS IN CYLINDRICAL DOMAINS
}

Abstract. We prove the large time existence of solutions to the magnetohydrodynamics equations with slip boundary conditions in a cylindrical domain. Assuming smallness of the $L_{2}$-norms of the derivatives of the initial velocity and of the magnetic field with respect to the variable along the axis of the cylinder, we are able to obtain an estimate for the velocity and the magnetic field in $W_{2}^{2,1}$ without restriction on their magnitude. Then the existence follows from the Leray-Schauder fixed point theorem.

1. Introduction. The aim of this paper is to prove the long time existence of solutions to the incompressible viscous magnetohydrodynamics equations (MHD). The equations determine the evolution of seven quantities: the velocity, the pressure and the magnetic field (see [6]). We assume the ideal slip boundary conditions for the velocity and the magnetic field.

We consider the following initial-boundary value problems:

$$
\begin{array}{ll}
v_{t}+v \cdot \nabla v-\operatorname{div} \mathbb{T}(v, p) & \\
\quad+H_{k} \cdot \nabla H_{k}-H \cdot \nabla H=0 & \text { in } \Omega^{T}=\Omega \times(0, T), \\
\operatorname{div} v=0, \operatorname{div} H=0 & \text { in } \Omega^{T}, \\
v \cdot \bar{n}=0 & \text { on } S^{T}=S \times(0, T), \\
\bar{n} \cdot \mathbb{T}(v, p) \cdot \bar{\tau}_{\alpha}=0, \quad \alpha=1,2, & \text { on } S^{T}, \\
\left.v\right|_{t=0}=v(0) & \text { in } \Omega,
\end{array}
$$

and

$$
\begin{array}{ll}
H_{t}+v \cdot \nabla H-H \cdot \nabla v-\nu_{\varkappa} \Delta H=0 & \text { in } \Omega^{T}, \\
H \cdot \bar{n}=0, \bar{n} \cdot \mathbb{D}(H) \cdot \bar{\tau}_{\alpha}=0, \quad \alpha=1,2, & \text { on } S^{T}, \\
\left.H\right|_{t=0}=H(0) & \text { in } \Omega,
\end{array}
$$

2010 Mathematics Subject Classification: 35Q35, 35G20, 35G30, 76W05.

Key words and phrases: incompressible magnetohydrodynamics, slip boundary conditions, large time existence, Leray-Schauder fixed point theorem. 
where $\Omega \subset \mathbb{R}^{3}$ is a cylindrical domain, $S=\partial \Omega$,

$$
v=v(x, t)=\left(v_{1}(x, t), v_{2}(x, t), v_{3}(x, t)\right) \in \mathbb{R}^{3}
$$

is the velocity of the magnetohydrodynamic fluid,

$$
H=H(x, t)=\left(H_{1}(x, t), H_{2}(x, t), H_{3}(x, t)\right) \in \mathbb{R}^{3}
$$

is the magnetic field, $p=p(x, t) \in \mathbb{R}^{1}$ denotes the pressure, $\bar{n}$ is the unit outward vector normal to the boundary $S$ and $\bar{\tau}_{\alpha}, \alpha=1,2$, are tangent vectors to $S$ and the dot denotes the scalar product in $\mathbb{R}^{3}$. Moreover, the summation convention over repeated indices is assumed. We define the stress tensor $\mathbb{T}(v, p)$ as

$$
\mathbb{T}(v, p)=\nu \mathbb{D}(v)-p I,
$$

where $\nu$ is the constant viscosity coefficient, $I$ the unit matrix and $\mathbb{D}(v)$ is the dilatation tensor of the form

$$
\mathbb{D}(v)=\left\{v_{i, x_{j}}+v_{j, x_{i}}\right\}_{i, j=1,2,3} .
$$

The equations contain two dissipative effects: dissipation of the mechanical energy

$$
\frac{\nu}{2} \int_{\Omega}|\mathbb{D}(v)|^{2} d x
$$

and dissipation of the magnetic energy

$$
\nu_{\varkappa} \int_{\Omega}|\operatorname{rot} H|^{2} d x
$$

where

$$
\nu_{\varkappa}=\frac{c^{2}}{4 \pi \sigma}
$$

is the Joule heat coefficient, $c$ is the light velocity, and $\sigma$ is the constant current conductivity.

The MHD equations describe the interaction between the motion of a conductive fluid and a magnetic field. The motion of a conductive fluid under a magnetic field generates electric fields and electric currents. The currents in the magnetic field evoke forces which on the one hand change the fluid motion and on the other hand alter the magnetic field. Therefore, the magnetic and hydrodynamic phenomena are strictly coupled.

Equations (1.1) describe the influence of a magnetic field on the fluid motion, while equations (1.2) determine generation of a magnetic field by the fluid motion.

By $x=\left(x_{1}, x_{2}, x_{3}\right)$ we denote the Cartesian coordinates; $\Omega \subset \mathbb{R}^{3}$ is a cylindrical type domain parallel to the $x_{3}$ axis with arbitrary cross section.

We assume that $S=S_{1} \cup S_{2}$ where $S_{1}$ is the part of the boundary which is parallel to the $x_{3}$ axis and $S_{2}$ is perpendicular to $x_{3}$. Hence 


$$
\begin{aligned}
& S_{1}=\left\{x \in \mathbb{R}^{3}: \varphi_{0}\left(x_{1}, x_{2}\right)=c_{0},-a<x_{3}<a\right\}, \\
& S_{2}=\left\{x \in \mathbb{R}^{3}: \varphi_{0}\left(x_{1}, x_{2}\right)<c_{0}, x_{3} \text { is either }-a \text { or } a\right\},
\end{aligned}
$$

where $a, c_{0}$ are given positive numbers and $\varphi_{0}\left(x_{1}, x_{2}\right)=c_{0}$ describes a sufficiently smooth closed curve in the plane $x_{3}=$ const.

Let us denote $h=v_{, x_{3}}, K=H_{, x_{3}}, w=v_{3}, \mu=H_{3}, \chi=(\operatorname{rot} v)_{3}$, $\theta=(\operatorname{rot} H)_{3}$ and define

$$
\begin{aligned}
R_{1} & =\|h(0)\|_{L_{2}(\Omega)}+\|K(0)\|_{L_{2}(\Omega)}+\|\chi(0)\|_{L_{2}(\Omega)}+\|\theta(0)\|_{L_{2}(\Omega)}, \\
R_{2} & =R_{1}+\zeta_{1}+\zeta_{2}+\|v(0)\|_{H^{1}(\Omega)}+\|H(0)\|_{H^{1}(\Omega)}, \\
R_{3} & =\|h(0)\|_{W_{\sigma}^{2-2 / \sigma}(\Omega)}+\|K(0)\|_{W_{\sigma}^{2-2 / \sigma}(\Omega)}, \\
d & =\|h(0)\|_{L_{2}(\Omega)}+\|K(0)\|_{L_{2}(\Omega)},
\end{aligned}
$$

where $\zeta_{1}, \zeta_{2}$ are introduced in (2.5) below. We prove the following result:

Theorem 1. Assume that $v(0), H(0), h(0)=v_{, x_{3}}(0), K(0)=H_{, x_{3}}(0)$, $\chi(0)=(\operatorname{rot} v)_{3}(0)$ belong to $L_{2}(\Omega)$, and $v(0), H(0)$ belong to $H^{1}(\Omega)$, while $h(0), K(0)$ belong to $W_{\sigma}^{2-2 / \sigma}(\Omega), 20 / 7<\sigma \leq 10 / 3$. Then there exists a solution to problem (1.1)-(1.2) such that $v \in W_{2}^{2,1}\left(\Omega^{T}\right), H \in W_{2}^{2,1}\left(\Omega^{T}\right)$, $\nabla p \in L_{2}\left(\Omega^{T}\right)$.

Moreover, if $q=p_{, x_{3}}$ and $5 / 3<\sigma<3$, then

$$
\begin{aligned}
& \|h\|_{W_{\sigma}^{2,1}\left(\Omega^{T}\right)}+\|K\|_{W_{\sigma}^{2,1}\left(\Omega^{T}\right)}+\|\nabla q\|_{L_{\sigma}\left(\Omega^{T}\right)}<L, \\
& \|v\|_{W_{2}^{2,1}\left(\Omega^{T}\right)}+\|\nabla p\|_{L_{2}\left(\Omega^{T}\right)}+\|H\|_{W_{2}^{2,1}\left(\Omega^{T}\right)} \leq \varphi\left(L, R_{2}\right),
\end{aligned}
$$

where $L$ is a constant chosen for a given $T$ so that, for an increasing function $\varphi$, sufficiently small constant $d$ and some constants $R_{i}, i=1,2,3$, involving the above norms,

$$
\varphi\left(L, R_{2}\right) d+c R_{3} \leq L \text { and } L>c R_{3},
$$

where $c$ is an absolute constant depending on imbedding only.

In this paper we extend the results of [8], 13] to the magnetohydrodynamic equations. The paper is organized in the following way. Using the estimate for the weak solutions to problem (1.1), (1.2) and assuming that $d$ is sufficiently small we prove a priori estimates (1.3) in Section 3. Applying the Leray-Schauder fixed point theorem, the existence of solutions in classes determined by (1.3) is proved in Section 4.

The paper starts the considerations concerning the existence of global regular large solutions to the magnetohydrodynamics equations. The next important step will be to examine a free boundary problem for large velocity and magnetic field. Hence we are going to generalize the results for small data from [2, 3, 4]. 


\section{Preliminaries}

2.1. Notation. The following function spaces will be used.

- Isotropic and anisotropic Lebesgue spaces:

$$
\begin{array}{ll}
L_{p}(Q), & Q \in\left\{\Omega^{T}, S^{T}, \Omega, S\right\}, p \in[1, \infty], \\
L_{q}\left(0, T ; L_{p}(Q)\right), & Q \in\{\Omega, S\}, p, q \in[1, \infty],
\end{array}
$$

with the norms

$$
\begin{aligned}
& \|u\|_{L_{p}(Q)}=|u|_{p, Q}, \\
& \|u\|_{L_{q}\left(0, T ; L_{p}(Q)\right)}=|u|_{p, q, Q^{T}} ;
\end{aligned}
$$

- Sobolev spaces:

$$
W_{q}^{s, s / 2}\left(Q^{T}\right), \quad Q \in\{S, \Omega\}, s \in \mathbb{Z}_{+} \cup\{0\}, q \in[1, \infty],
$$

with the norm

$$
\|u\|_{W_{q}^{s, s / 2}\left(Q^{T}\right)}=\left(\sum_{|\alpha|+2 i \leq s} \int_{Q^{T}}\left|D_{x}^{\alpha} \partial_{t}^{i} u\right|^{q} d x d t\right)^{1 / q},
$$

where

$$
D_{x}^{\alpha}=\partial_{x_{1}}^{\alpha_{1}} \partial_{x_{2}}^{\alpha_{2}} \partial_{x_{3}}^{\alpha_{3}}, \quad|\alpha|=\alpha_{1}+\alpha_{2}+\alpha_{3}, i, \alpha_{j} \in \mathbb{Z}_{+} \cup\{0\} .
$$

In the special case $q=2$,

$$
H^{s}(Q)=W_{2}^{s}(Q), \quad Q \in\{S, \Omega\}, s \in \mathbb{Z}_{+} \cup\{0\}, q \in[1, \infty],
$$

with the norm

$$
\|u\|_{H^{s}(Q)}=\left(\sum_{|\alpha| \leq s} \int_{Q}\left|D_{x}^{\alpha} u\right|^{2} d x\right)^{1 / 2} .
$$

We define a space natural for weak solutions to the Navier-Stokes and MHD equations:

$$
\begin{aligned}
V_{2}^{k}\left(\Omega^{T}\right)=\left\{\varphi:\|\varphi\|_{V_{2}^{k}\left(\Omega^{T}\right)}=\underset{t \in(0, T)}{\operatorname{esssup}}\|\varphi\|_{H^{k}(\Omega)}\right. & \\
& \left.+\left(\int_{0}^{T}\|\nabla \varphi\|_{H^{k}(\Omega)}^{2} d t\right)^{1 / 2}<\infty\right\}
\end{aligned}
$$

with $k \in \mathbb{N}$, and $L_{2}$ replaces $H^{0}$ in the definition of $V_{2}^{0}$.

For simplicity we define

$$
\|a, b\|_{X}=\|a\|_{X}+\|b\|_{X}, \quad\|A\|_{X}=\|v\|_{X}+\|H\|_{X}, \quad|a|_{s, \Omega}=\|a\|_{L_{s}(\Omega)},
$$

where $A=(v, H)$. 
2.2. Weak solutions. By weak solutions to problem (1.1) we mean functions $v, H$ satisfying the integral identities

$$
\begin{aligned}
-\int_{\Omega^{T}} v \cdot \varphi_{t} d x d t+\int_{\Omega^{T}} \mathbb{D}(v) \cdot \mathbb{D}(\varphi) d x d t+\int_{\Omega^{T}} H_{i} H_{j} & \nabla_{i} \varphi_{j} d x d t \\
& =\int_{\Omega} v(0) \cdot \varphi(0) d x, \\
-\int_{\Omega^{T}} H \cdot \psi_{t} d x d t+\int_{\Omega^{T}} \mathbb{D}(H) \cdot \mathbb{D}(\psi) d x d t+\int_{\Omega^{T}}\left(v_{j} H_{i}\right. & \left.-v_{i} H_{j}\right) \nabla_{i} \psi_{j} d x d t \\
& =\int_{\Omega} H(0) \cdot \psi(0) d x,
\end{aligned}
$$

which hold for any functions $\varphi, \psi \in W_{2}^{1,1}\left(\Omega^{T}\right)$ such that $\left.\varphi \cdot \bar{n}\right|_{S}=0,\left.\psi \cdot \bar{n}\right|_{S}$ $=0, \varphi(T)=0, \psi(T)=0$. Moreover, weak solutions $v$ and $H$ must satisfy the boundary conditions

$$
\begin{array}{ll}
\left.v \cdot \bar{n}\right|_{S}=0, & \left.H \cdot \bar{n}\right|_{S}=0, \\
\left.\bar{n} \cdot \mathbb{D}(v) \cdot \bar{\tau}_{\alpha}\right|_{S}=0, & \left.\bar{n} \cdot \mathbb{D}(H) \cdot \bar{\tau}_{\alpha}\right|_{S}=0, \quad \alpha=1,2 .
\end{array}
$$

Finally, the summation convention over repeated indices is assumed and

$$
\mathbb{D}(k) \cdot \mathbb{D}(\chi)=d_{i j}(k) d_{i j}(\chi)
$$

where $d_{i j}(k)=k_{i, x_{j}}+k_{j, x_{i}}$, with $k \in\{v, H, \chi-\varphi, \psi\}$.

For the weak solutions we have the Korn inequality:

Lemma 2.1. Assume that

$$
\begin{aligned}
& E_{\Omega}(v, H):=\|\mathbb{D}(v)\|_{L_{2}(\Omega)}^{2}+\|\mathbb{D}(H)\|_{L_{2}(\Omega)}^{2}<\infty, \\
& \left.v \cdot \bar{n}\right|_{S}=0, \quad \operatorname{div} v=0, \quad \operatorname{div} H=0,\left.\quad H \cdot \bar{n}\right|_{S}=0 .
\end{aligned}
$$

Assume that $\Omega$ is not axially symmetric. Then there exists a constant $c_{1}$ such that

$$
\|A\|_{H^{1}(\Omega)}^{2} \leq c_{1} E_{\Omega}(v, H) .
$$

If $\Omega$ is axially symmetric, $\eta=\left(-x_{2}, x_{1}, 0\right)$, then there exists a constant $c_{2}$ such that

$$
\|A\|_{H^{1}(\Omega)}^{2} \leq c_{2}\left(E_{\Omega}(v, H)+v_{\eta}^{2}+H_{\eta}^{2}\right) .
$$

where $v_{\eta}=\int_{\Omega} v \cdot \eta d x, H_{\eta}=\int_{\Omega} h \cdot \eta d x$.

Proof. Let $u$ be a function such that

$$
E_{\Omega}(u)<\infty, \quad \operatorname{div} u=0,\left.\quad u \cdot \bar{n}\right|_{S}=0 .
$$

Assume that $\Omega$ is not axially symmetric. Then

$$
E_{\Omega}(u)=2\|\nabla u\|_{L_{2}(\Omega)}^{2}+2 \int_{\Omega} \partial_{x_{i}} u_{j} \partial_{x_{j}} u_{i} d x,
$$


where the last term equals $2 \int_{S} \partial_{x_{i}} n_{j} u_{i} u_{j} d S$ so it is bounded by $c\|u\|_{L_{2}(S)}^{2}$. Hence we get

$$
\|\nabla u\|_{L_{2}(\Omega)}^{2} \leq c\left(E_{\Omega}(u)+\|u\|_{L_{2}(S)}^{2}\right) .
$$

By interpolation we have

$$
\|\nabla u\|_{L_{2}(\Omega)}^{2} \leq c\left(E_{\Omega}(u)+\|u\|_{L_{2}(\Omega)}^{2}\right) .
$$

By [9] there exist constants $\delta, M$, where $\delta$ can be chosen arbitrarily small, such that

$$
\|u\|_{L_{2}(\Omega)}^{2} \leq \delta\|\nabla u\|_{L_{2}(\Omega)}^{2}+M E_{\Omega}(u) .
$$

Then we obtain

$$
\|u\|_{H^{1}(\Omega)} \leq c E_{\Omega}(u) .
$$

Replacing $u$ by $v$ and $H$ we prove the lemma in the case of $\Omega$ non-axially symmetric.

Let $\Omega$ be axially symmetric, with the $x_{3}$ axis being the axis of symmetry. Let $u=u^{\prime}+u_{\eta} \eta /\|\eta\|_{L_{2}(\Omega)}^{2} \equiv u^{\prime}+u^{\prime \prime}$, where $\int_{\Omega} u^{\prime} \cdot \eta d x=0, u_{\eta}=\int_{\Omega} u \cdot \eta d x$. Then $E_{\Omega}\left(u^{\prime \prime}\right)=0,\left.u^{\prime \prime} \cdot \bar{n}\right|_{S}=0$.

From the non-axially symmetric case we obtain

$$
\left\|u^{\prime}\right\|_{H^{1}(\Omega)}^{2} \leq c E_{\Omega}\left(u^{\prime}\right)
$$

Hence

$$
\|u\|_{H^{1}(\Omega)}^{2} \leq c\left(E_{\Omega}(u)+\left|u_{\eta}\right|^{2}\right) .
$$

Since $u$ can be replaced by $v$ and $H$ we obtain (2.4). This concludes the proof.

Now we formulate energy type estimates for weak solutions of (1.1) and (1.2).

Lemma 2.2. Let $v(0), H(0) \in L_{2}(\Omega)$. Let $T>0$ be given. Then there exist constants

$$
\begin{aligned}
& \zeta_{1}^{2}=\|v(0)\|_{L_{2}(\Omega)}^{2}+\|H(0)\|_{L_{2}(\Omega)}^{2}, \\
& \zeta_{2}^{2}=c_{4} \zeta_{1}^{2}, \\
& \zeta_{3}^{2}=c_{7} e^{c_{6} T} \zeta_{1}^{2},
\end{aligned}
$$

where $c_{4}=1 / \min \left\{1, c_{3}\right\}, c_{3}=\min \left\{\nu / c_{1}, \nu_{\varkappa} / c_{1}\right\}$ and $c_{1}$ appears in (2.3), $c_{7}=1 / \min \left\{1, c_{5}\right\}, c_{5}=\min \left\{\nu / c_{2}, \nu_{\varkappa} / c_{2}\right\}, c_{6}=\|\eta\|_{L_{2}(\Omega)}^{2}$, which do not depend on $k_{0}=k T, k \in \mathbb{N}$, such that in the non-axially symmetric case we have

$$
\begin{array}{ll}
\|v(t)\|_{L_{2}(\Omega)}+\|H(t)\|_{L_{2}(\Omega)} \leq \zeta_{1} & \text { for any } t \geq 0, \\
\|H, v\|_{V_{2}^{0}(\Omega \times(k T, t))} \leq \zeta_{2} & \text { for } t \in(k T,(k+1) T), k \in \mathbb{N},
\end{array}
$$


and in the axially symmetric case

$$
\begin{array}{ll}
|v(t), H(t)|_{2, \Omega} \leq \zeta_{1} \quad \text { for any } t \geq 0, \\
\|v, H\|_{V_{2}^{0}(\Omega \times(k T, t))} \leq \zeta_{3} & \text { for } t \in(k T,(k+1) T), k \in \mathbb{N} .
\end{array}
$$

Proof. Multiplying $(1.1)_{1}$ by $v,(1.2)_{1}$ by $H$, integrating over $\Omega$ and using the boundary conditions we obtain

$$
\frac{1}{2} \frac{d}{d t}\left(\|v\|_{L_{2}(\Omega)}^{2}+\|H\|_{L_{2}(\Omega)}^{2}\right)+\frac{c_{3}}{2}\left(\|v\|_{H^{1}(\Omega)}^{2}+\|H\|_{H^{1}(\Omega)}^{2}\right) \leq 0,
$$

where $c_{3}=\min \left\{\nu / c_{1}, \nu_{\varkappa} / c_{1}\right\}$.

Omitting the terms with viscosity coefficients and integrating the result with respect to time yields

$$
\|v(t)\|_{L_{2}(\Omega)}^{2}+\|H(t)\|_{L_{2}(\Omega)}^{2} \leq\|v(0)\|_{L_{2}(\Omega)}^{2}+\|H(0)\|_{L_{2}(\Omega)}^{2} \equiv \zeta_{1}^{2},
$$

so $(2.6)_{1}$ holds.

Integrating (2.8) with respect to time from $k T$ to $t \in(k T,(k+1) T]$, $k \in \mathbb{N}_{0}$, we obtain

$$
\|A\|_{V_{2}^{0}(\Omega \times(k T, t))}^{2} \leq c_{4}\left(\|v(k T)\|_{L_{2}(\Omega)}^{2}+\|H(k T)\|_{L_{2}(\Omega)}^{2}\right) \leq c_{4} \zeta_{1}^{2},
$$

where $(2.9)$ was used and $c_{4}=1 / \min \left\{1, c_{3}\right\}$.

Let us consider the axially symmetric case. Multiplying $(1.1)_{1}$ by $v,(1.2)_{1}$ by $H$, integrating over $\Omega$ and using the boundary conditions we obtain

$$
\frac{d}{d t}\left(\|v\|_{L_{2}(\Omega)}^{2}+\|H\|_{L_{2}(\Omega)}^{2}\right)+\nu\|\mathbb{D}(v)\|_{L_{2}(\Omega)}^{2}+\nu_{\varkappa}\|\mathbb{D}(H)\|_{L_{2}(\Omega)}^{2} \leq 0 .
$$

Omitting the last two terms on the l.h.s. and integrating the result with respect to time we obtain

$$
\|v(t)\|_{L_{2}(\Omega)}^{2}+\|H(t)\|_{L_{2}(\Omega)}^{2} \leq\|v(0)\|_{L_{2}(\Omega)}^{2}+\|H(0)\|_{L_{2}(\Omega)}^{2} .
$$

In view of the Korn inequality (2.4) we obtain from (2.11) the inequality

$$
\begin{array}{r}
\frac{d}{d t}\left(\|v\|_{L_{2}(\Omega)}^{2}+\|H\|_{L_{2}(\Omega)}^{2}\right)+c_{5}\left(\|v\|_{H^{1}(\Omega)}^{2}+\|H\|_{H^{1}(\Omega)}^{2}\right) \\
\quad \leq\left|v_{\eta}\right|^{2}+\left|H_{\eta}\right|^{2} \leq c_{6}\left(\|v\|_{L_{2}(\Omega)}^{2}+\|H\|_{L_{2}(\Omega)}^{2}\right),
\end{array}
$$

where $c_{5}=\min \left\{\nu / c_{2}, \nu_{\varkappa} / c_{2}\right\}, c_{6}=\|\eta\|_{L_{2}(\Omega)}^{2}$.

From (2.13) we have

$$
\begin{aligned}
\frac{d}{d t}\left[\left(\|v\|_{L_{2}(\Omega)}^{2}+\|H\|_{L_{2}(\Omega)}^{2}\right) e^{-c_{6} t}\right] & \\
& +c_{5}\left(\|v\|_{H^{1}(\Omega)}^{2}+\|H\|_{H^{1}(\Omega)}^{2}\right) e^{-c_{6} t} \leq 0 .
\end{aligned}
$$

Integrating (2.13) with respect to time from $k T$ to $t \in(k T,(k+1) T)$ yields 


$$
\begin{gathered}
\left(\|v(t)\|_{H^{1}(\Omega)}^{2}+\right. \\
\left.\quad H(t) \|_{H^{1}(\Omega)}^{2}\right) e^{-c_{6} t} \\
\quad+c_{5} \int_{k T}^{t}\left(\left\|v\left(t^{\prime}\right)\right\|_{H^{1}(\Omega)}^{2}+\left\|H\left(t^{\prime}\right)\right\|_{H^{1}(\Omega)}^{2}\right) e^{-c_{6} t^{\prime}} d t^{\prime} \\
\leq\left(\|v(k T)\|_{L_{2}(\Omega)}^{2}+\|H(k T)\|_{L_{2}(\Omega)}^{2}\right) e^{-c_{6} k T} .
\end{gathered}
$$

Multiplying (2.15) by $e^{c_{6} t}$ and using (2.12) we obtain

$$
\begin{gathered}
\|v(t)\|_{L_{2}(\Omega)}^{2}+\|H(t)\|_{L_{2}(\Omega)}^{2}+c_{5} e^{c_{6} t} \int_{k T}^{t}\left(\left\|v\left(t^{\prime}\right)\right\|_{H^{1}(\Omega)}^{2}+\left\|H\left(t^{\prime}\right)\right\|_{H^{1}(\Omega)}^{2}\right) e^{-c_{6} t^{\prime}} d t^{\prime} \\
\leq\left(\|v(k T)\|_{L_{2}(\Omega)}^{2}+\|H(k T)\|_{L_{2}(\Omega)}^{2}\right) e^{c_{6}(t-k T)} \\
\leq\left(\|v(0)\|_{L_{2}(\Omega)}^{2}+\|H(0)\|_{L_{2}(\Omega)}^{2}\right) e^{c_{6} T} .
\end{gathered}
$$

From (2.16) we obtain $(2.7)_{2}$, and (2.12) yields $(2.7)_{1}$. This concludes the proof.

From the above lemma by an application of the Galerkin method and the considerations from [5, Ch. 6], we have

Lemma 2.3. Let the assumptions of Lemma 2.2 hold. Then there exists a weak solution to problems (1.1) and (1.2) in any interval $(k T,(k+1) T)$, $k \in \mathbb{N}$, satisfying

$$
\|v, H\|_{V_{2}^{0}(\Omega \times(k T,(k+1) T)} \leq \zeta_{i},
$$

where $i=2$ for a non-axially symmetric domain and $i=4$ for an axially symmetric domain.

2.3. Auxiliary problems. In this paper the non-axially symmetric case is examined. We distinguish the direction $x_{3}$. In order to derive estimates for derivatives in direction $x_{3}$ we introduce the quantities

$$
h=v_{, x_{3}}, \quad K=H_{, x_{3}}, \quad q=p_{, x_{3}} .
$$

These functions are solutions to the problems listed below

Lemma 2.4. The pair of functions $(h, q)$ is a solution to the problem

$$
\begin{array}{ll}
h_{t}-\nu \Delta h+\nabla q=K \cdot \nabla H+H \cdot \nabla K-v \cdot \nabla h & \\
\quad-h \cdot \nabla v-K_{i} \cdot \nabla H_{i}-H_{i} \cdot \nabla K_{i} & \text { in } \Omega^{T}, \\
\operatorname{div} h=0 & \text { in } \Omega^{T}, \\
h \cdot \bar{n}=0, \bar{n} \cdot \mathbb{D}(h) \cdot \bar{\tau}_{\alpha}=0, \quad \alpha=1,2, & \text { on } S_{1}^{T}, \\
h_{i}=0, \quad i=1,2, \quad h_{3, x_{3}}=0 & \text { on } S_{2}^{T}, \\
\left.h\right|_{t=0}=h(0) & \text { in } \Omega,
\end{array}
$$

where $v, H, K$ are given. 
Lemma 2.5. The function $K$ is a solution to the problem

$$
\begin{array}{ll}
K_{t}-\nu_{\varkappa} \Delta K=K \cdot \nabla v+H \cdot \nabla h-h \cdot \nabla H-v \cdot \nabla K & \text { in } \Omega^{T}, \\
K \cdot \bar{n}=0, \quad \bar{n} \cdot \mathbb{D}(K) \cdot \bar{\tau}_{\alpha}=0, \quad \alpha=1,2, & \text { on } S_{1}^{T}, \\
K_{j}=0, \quad j=1,2, \quad K_{3, x_{3}}=0 & \text { on } S_{2}^{T}, \\
\left.K\right|_{t=0}=K(0) & \text { in } \Omega,
\end{array}
$$

where $v, h, H$ are treated as given.

We use the following estimates for $h$ and $K$ (see [8], [11], [13]).

Lemma 2.6. Assume that pair of function $(v, H)$ is a weak solution to problems (1.1) and (1.2). Assume that $h \in L_{\infty}\left(0, T ; L_{3}(\Omega)\right), K \in L_{\infty}(0, T$; $\left.L_{3}(\Omega)\right), h(0) \in L_{2}(\Omega), K(0) \in L_{2}(\Omega)$. Then

$$
\|h, K\|_{V_{2}^{0}\left(\Omega^{T}\right)}^{2} \leq c\left(\zeta_{2}^{2}\|h, K\|_{L_{\infty}\left(0, t ; L_{3}(\Omega)\right)}^{2}+|h(0), K(0)|_{2, \Omega}^{2}\right)
$$

for all $t \leq T$.

LEMma 2.7. With $h(0)$ and $K(0)$ as in the previous lemma and $\nabla v, \nabla H$ $\in L_{2}\left(0, t ; L_{3}(\Omega)\right)$, every weak solution to (1.1) and (1.2) satisfies

$$
\begin{aligned}
\|h(t), K(t)\|_{V_{2}^{0}\left(\Omega^{t}\right)}^{2} \leq & c \exp \left\{c\left(\|\nabla v\|_{L_{2}\left(0, t ; L_{3}(\Omega)\right)}^{2}+\|\nabla H\|_{L_{2}\left(0, t ; L_{3}(\Omega)\right)}^{2}\right)\right\} \\
& \cdot\left(|h(0)|_{2, \Omega}^{2}+|K(0)|_{2, \Omega}^{2}\right), \quad t \leq T .
\end{aligned}
$$

Lemma 2.8. Let $q, v, H, K$ be given. Let $\mu=H_{3}$. Then $w=v_{3}$ is a solution to the problem

$$
\begin{array}{ll}
w_{t}+v \cdot \nabla w-\nu \Delta w+q-H \cdot \nabla \mu+H \cdot K=0 & \text { in } \Omega^{T}, \\
w_{, n}=0 & \text { on } S_{1}^{T}, \\
w=0 & \text { on } S_{2}^{T}, \\
\left.w\right|_{t=0}=w(0) & \text { in } \Omega .
\end{array}
$$

Lemma 2.9. Let $v, H$ be given. Let $\mu=H_{3}$. Then $\mu$ is a solution to the problem

$$
\begin{array}{ll}
\mu_{t}+v \cdot \nabla \mu-H \cdot \nabla w-\nu_{\varkappa} \Delta \mu=0 & \text { in } \Omega^{T}, \\
\mu_{, n}=0 & \text { on } S_{1}^{T}, \\
\mu=0 & \text { on } S_{2}^{T}, \\
\left.\mu\right|_{t=0}=\mu(0) & \text { in } \Omega .
\end{array}
$$

Lemma 2.10. Let $h, k, v, H, w, \mu$ be given. Then $\chi=(\operatorname{rot} v)_{3}$ is a solution to the problem 


$$
\begin{aligned}
& \chi, t & +v \cdot \nabla \chi-h_{3} \chi+w_{, x_{1}} h_{2}-h_{1} w_{, x_{2}}+K_{3} \theta-K_{2} \mu_{, x_{1}} & \\
& +\mu_{, x_{2}} K_{1}-H \cdot \nabla \theta-\nu \Delta \chi=0 & & \text { in } \Omega^{T}, \\
(2.23) & \chi=v_{i}\left(n_{i, x_{j}} \tau_{1 j}+\tau_{1 i, x_{j}} n_{j}\right)+v \cdot \bar{\tau}_{1}\left(\tau_{12, x_{1}}-\tau_{11, x_{2}}\right) \equiv \chi_{*} & & \text { on } S_{1}^{T}, \\
& \chi \chi_{, x_{3}}=0 & & \text { on } S_{2}^{T}, \\
& \left.\chi\right|_{t=0}=\chi(0) & & \text { in } \Omega,
\end{aligned}
$$

and $\theta=(\operatorname{rot} H)_{3}$ is a solution to the problem

$$
\begin{aligned}
& \theta_{, t}+v \cdot \nabla \theta-h_{3} \theta-K_{3} \chi+w_{, x_{1}} K_{2}-w_{, x_{2}} K_{1}+\mu_{, x_{1}} h_{2} \\
& -\mu_{, x_{2}} h_{1}-H \cdot \nabla \chi-\nu_{\varkappa} \Delta \theta=0 \quad \text { in } \Omega^{T}, \\
& \theta=H_{i}\left(n_{i, x_{j}} \tau_{1 j}+\tau_{1 i, x_{j}} n_{j}\right)+H \cdot \bar{\tau}_{1}\left(\tau_{12, x_{1}}-\tau_{11, x_{2}}\right)=\theta_{*} \quad \text { on } S_{1}^{T} \text {, } \\
& \theta_{, x_{3}}=0 \quad \text { on } S_{2}^{T} \text {, } \\
& \left.\theta\right|_{t=0}=\theta(0) \quad \text { in } \Omega \text {, }
\end{aligned}
$$

where

$$
\begin{aligned}
& \left.\bar{n}\right|_{S_{1}}=\frac{\nabla \varphi}{|\nabla \varphi|}=\frac{1}{|\nabla \varphi|}\left(\varphi, x_{1}, \varphi_{, x_{2}}, 0\right), \\
& \left.\bar{\tau}_{1}\right|_{S_{1}}=\frac{\nabla^{\perp} \varphi}{|\nabla \varphi|}=\frac{1}{|\nabla \varphi|}\left(-\varphi, x_{2}, \varphi, x_{1}, 0\right),\left.\quad \bar{\tau}_{2}\right|_{S_{1}}=(0,0,1), \\
& \left.\bar{n}\right|_{S_{2}}=(0,0,1),\left.\quad \bar{\tau}_{1}\right|_{S_{2}}=(1,0,0),\left.\quad \bar{\tau}_{2}\right|_{S_{2}}=(0,1,0) .
\end{aligned}
$$

3. Estimates. Let us introduce the function $\tilde{\chi}$ as a solution to the problem

$$
\begin{array}{ll}
\tilde{\chi}_{, t}-\nu \Delta \tilde{\chi}=0 & \text { in } \Omega^{T}, \\
\tilde{\chi}=\chi_{*} & \text { on } S_{1}^{T}, \\
\tilde{\chi}_{, x_{3}}=0 & \text { on } S_{2}^{T}, \\
\left.\tilde{\chi}\right|_{t=0}=0 & \text { in } \Omega .
\end{array}
$$

Then the new function

$$
\chi^{\prime}=\chi-\tilde{\chi}
$$

is a solution to the problem

$$
\begin{array}{lr}
\chi_{, t}^{\prime}+v \cdot \nabla \chi^{\prime}-\nu \Delta \chi^{\prime}-h_{3} \chi^{\prime}-h_{1} w_{, x_{2}}+h_{2} w_{, x_{1}} & \\
\quad+K_{1} \mu_{x_{2}}-K_{2} \mu_{x_{1}}+K_{3} \theta-H \cdot \nabla \theta=h_{3} \tilde{\chi}-v \cdot \nabla \tilde{\chi} & \text { in } \Omega^{T}, \\
\chi^{\prime}=0 & \text { on } S_{1}^{T}, \\
\chi_{, x_{3}}^{\prime}=0 & \text { on } S_{2}^{T}, \\
\left.\chi^{\prime}\right|_{t=0}=\chi(0) & \text { in } \Omega .
\end{array}
$$


Let $\tilde{\theta}$ be a solution to the problem

$$
\begin{array}{ll}
\tilde{\theta}_{, t}-\nu_{\varkappa} \Delta \tilde{\theta}=0 & \text { in } \Omega^{T}, \\
\tilde{\theta}=\theta_{*} & \text { on } S_{1}^{T}, \\
\tilde{\theta}_{x_{3}}=0 & \text { on } S_{2}^{T}, \\
\left.\tilde{\theta}\right|_{t=0}=0 & \text { in } \Omega .
\end{array}
$$

Then the function

$$
\theta^{\prime}=\theta-\tilde{\theta}
$$

is a solution to the problem

$$
\begin{aligned}
& \theta_{, t}^{\prime}+v \cdot \nabla \theta^{\prime}-h_{3} \theta^{\prime}-K_{3} \chi+w_{, x_{1}} K_{2}-w_{x_{2}} K_{1} \\
& +\mu_{, x_{1}} h_{2}-\mu_{, x_{2}} h_{1}-H \cdot \nabla \chi-\nu_{\varkappa} \Delta \theta^{\prime}=h_{3} \tilde{\theta}-v \cdot \nabla \tilde{\theta} \quad \text { in } \Omega^{T}, \\
& \theta^{\prime}=0 \quad \text { on } S_{1}^{T} \text {, } \\
& \theta_{, x_{3}}^{\prime}=0 \quad \text { on } S_{2}^{T} \text {, } \\
& \left.\theta^{\prime}\right|_{t=0}=\theta(0) \quad \text { in } \Omega \text {. }
\end{aligned}
$$

Let $\eta=(\chi, \theta)$ and $|\eta|_{X} \equiv|\chi|_{X}+|\theta|_{X}$.

Lemma 3.1. Assume that $v^{\prime}=\left(v_{1}, v_{2}\right) \in L_{\infty}\left(0, T ; L_{3}(S)\right) \cap H^{1 / 2+\varepsilon}\left(\Omega^{T}\right) \cap W_{2}^{1,1 / 2}\left(\Omega^{T}\right)$, $h \in L_{\infty}\left(0, T ; L_{3}(\Omega)\right), \quad K \in L_{\infty}\left(0, T ; L_{3}(\Omega)\right), \quad \chi(0) \in L_{2}(\Omega), \quad \theta(0) \in L_{2}(\Omega)$, $H^{\prime}=\left(H_{1}, H_{2}\right) \in L_{\infty}\left(0, T ; L_{3}(S)\right) \cap H^{1 / 2+\varepsilon}(\Omega) \cap W_{2}^{1,1 / 2}\left(\Omega^{T}\right)$.

Let the assumptions of Lemma 2.2 be satisfied. Then solutions of problems (2.23) and (2.24) satisfy

$$
\begin{aligned}
& \|\eta\|_{V_{2}^{0}\left(\Omega^{t}\right)}^{2} \leq c\left[\zeta_{2}^{2}\|h, K\|_{L_{\infty}\left(0, t ; L_{3}(\Omega)\right)}^{2}+\left\|A^{\prime}\right\|_{L_{\infty}\left(0, t ; L_{3}(S)\right)}^{2}\right. \\
& \left.\quad+\left\|A^{\prime}\right\|_{L_{\infty}\left(0, t ; H^{1 / 2+\varepsilon}(\Omega)\right)}^{2}+\left\|A^{\prime}\right\|_{W_{2}^{1,1 / 2}\left(\Omega^{t}\right)}^{2}+|\eta(0)|_{2, \Omega}^{2}\right], \quad t \leq T,
\end{aligned}
$$

for all $\varepsilon>0$, where $A^{\prime}=\left(v^{\prime}, H^{\prime}\right)$.

Proof. Multiplying $(3.2)_{1}$ by $\chi^{\prime}$, integrating over $\Omega$, and using the boundary conditions $(3.2)_{2,3}$ we obtain

$$
\begin{gathered}
\frac{1}{2} \frac{d}{d t}\left\|\chi^{\prime}\right\|_{L_{2}(\Omega)}^{2}+\nu\left|\nabla \chi^{\prime}\right|_{2, \Omega}^{2}=\int_{\Omega} h_{3} \chi^{\prime 2} d x-\int_{\Omega}\left(h_{2} w_{, x_{1}}-h_{1} w_{, x_{2}}\right) \chi^{\prime} d x \\
-\int_{\Omega} v \cdot \nabla \tilde{\chi} \chi^{\prime} d x+\int_{\Omega} h_{3} \tilde{\chi} \chi^{\prime} d x+\int_{\Omega}\left(K_{2} \mu_{, x_{1}}-K_{1} \mu_{, x_{2}}\right) \chi^{\prime} d x \\
-\int_{\Omega} K_{3} \theta \chi^{\prime} d x+\int_{\Omega} H \cdot \nabla \theta \chi^{\prime} d x=\sum_{k=1}^{7} I_{k} .
\end{gathered}
$$


Now we estimate the terms on the r.h.s. of the above equality. First,

$$
\begin{aligned}
I_{1} & =\int_{\Omega} h_{3} \chi^{\prime 2} d x \leq \varepsilon_{1}\left|\chi^{\prime}\right|_{6, \Omega}^{2}+c\left(1 / \varepsilon_{1}\right)\left|\chi^{\prime}\right|_{2, \Omega}^{2}|h|_{3, \Omega}^{2} \\
& \leq \varepsilon_{1}\left|\chi^{\prime}\right|_{6, \Omega}^{2}+c\left(1 / \varepsilon_{1}\right)\left(|\tilde{\chi}|_{2, \Omega}^{2}+|\chi|_{2, \Omega}^{2}\right)|h|_{3, \Omega}^{2} .
\end{aligned}
$$

The other terms on the r.h.s. of (3.6) can be estimated by

$$
\begin{aligned}
& I_{2} \leq \frac{\varepsilon_{2}}{2}\left|\chi^{\prime}\right|_{6, \Omega}^{2}+\frac{1}{2 \varepsilon_{2}}\left|w_{, x^{\prime}}\right|_{2, \Omega}^{2}|h|_{3, \Omega}^{2}, \\
& I_{3} \leq \varepsilon_{3}\left|\nabla \chi^{\prime}\right|_{2, \Omega}^{2}+c\left(1 / \varepsilon_{3}\right)|v|_{6, \Omega}^{2}|\tilde{\chi}|_{3, \Omega}^{2}, \\
& I_{4} \leq \varepsilon_{4}\left|\chi^{\prime}\right|_{6, \Omega}^{2}+c\left(1 / \varepsilon_{4}\right)|h|_{12 / 7, \Omega}^{2}|\tilde{\chi}|_{4, \Omega}^{2}, \\
& I_{5} \leq \varepsilon_{5}\left|\chi^{\prime}\right|_{6, \Omega}^{2}+c\left(1 / \varepsilon_{5}\right)|K|_{3, \Omega}^{2}\left|\mu_{, x^{\prime}}\right|_{2, \Omega}^{2}, \\
& I_{6} \leq \varepsilon_{6}\left|\chi^{\prime}\right|_{6, \Omega}^{2}+c\left(1 / \varepsilon_{6}\right)|K|_{3, \Omega}^{2}|\theta|_{2, \Omega}^{2} .
\end{aligned}
$$

This implies that

$$
\begin{aligned}
\frac{1}{2} \frac{d}{d t}\left|\chi^{\prime}\right|_{2, \Omega}^{2}+ & \nu\left|\nabla \chi^{\prime}\right|_{2, \Omega}^{2} \leq c\left[|h|_{3, \Omega}^{2}\left|w_{, x^{\prime}}\right|_{2, \Omega}^{2}\right. \\
& +\left(|\chi|_{2, \Omega}^{2}+|\tilde{\chi}|_{2, \Omega}^{2}\right)|h|_{3, \Omega}^{2}+|\tilde{\chi}|_{3, \Omega}^{2}|v|_{6, \Omega}^{2}+|h|_{12 / 7, \Omega}^{2}|\tilde{\chi}|_{4, \Omega}^{2} \\
& \left.+|K|_{3, \Omega}^{2}\left|\mu_{, x^{\prime}}\right|_{2, \Omega}^{2}+|K|_{3, \Omega}^{2}|\theta|_{2, \Omega}^{2}\right]+\int_{\Omega} H \cdot \nabla \theta \chi^{\prime} d x .
\end{aligned}
$$

Next multiplying (3.4) 1 by $\theta^{\prime}$, integrating over $\Omega$, and using the boundary conditions $(3.4)_{2,3}$ we obtain

$$
\begin{aligned}
\frac{1}{2} \frac{d}{d t}\left|\theta^{\prime}\right|_{2, \Omega}^{2}+\nu_{\varkappa} \mid & \left.\nabla \theta^{\prime}\right|_{2, \Omega} ^{2} \leq \int_{\Omega}\left|h_{3} \theta^{\prime 2}\right| d x+\int_{\Omega}\left|K_{3} \chi \theta^{\prime}\right| d x \\
& +\int_{\Omega}\left|K_{1} w_{x_{2}} \theta^{\prime}\right| d x+\int_{\Omega}\left|K_{2} w_{, x_{1}} \theta^{\prime}\right| d x+\int_{\Omega}\left|h_{2} \mu_{, x_{1}} \theta^{\prime}\right| d x \\
& +\int_{\Omega}\left|\mu_{, x_{2}} h_{1} \theta^{\prime}\right| d x+\int_{\Omega} H \cdot \nabla \chi \theta^{\prime} d x+\int_{\Omega}\left|h_{3} \tilde{\theta} \theta^{\prime}\right| d x \\
& +\int_{\Omega}\left|v \cdot \nabla \theta^{\prime} \tilde{\theta}\right| d x^{\prime}=\sum_{j=1}^{9} J_{j} .
\end{aligned}
$$

Now we estimate the integrals on the r.h.s. of (3.8):

$$
\begin{aligned}
& J_{1} \leq \varepsilon_{1}\left|\theta^{\prime}\right|_{6, \Omega}^{2}+c\left(1 / \varepsilon_{1}\right)\left|\theta^{\prime}\right|_{2, \Omega}^{2}|h|_{3, \Omega}^{2}, \\
& J_{2} \leq \varepsilon_{2}\left|\theta^{\prime}\right|_{6, \Omega}^{2}+c\left(1 / \varepsilon_{2}\right)|K|_{3, \Omega}^{2}|\chi|_{2, \Omega}^{2}, \\
& J_{3}+J_{4} \leq \varepsilon_{3}\left|\theta^{\prime}\right|_{6, \Omega}^{2}+c\left(1 / \varepsilon_{3}\right)|K|_{3, \Omega}^{2}\left|w_{, x^{\prime}}\right|_{2, \Omega}^{2}, \\
& J_{5}+J_{6} \leq \varepsilon_{4}\left|\theta^{\prime}\right|_{6, \Omega}^{2}+c\left(1 / \varepsilon_{4}\right)|h|_{3, \Omega}^{2}\left|\mu_{, x^{\prime}}\right|_{2, \Omega}^{2}, \\
& J_{8} \leq \varepsilon_{5}\left|\theta^{\prime}\right|_{6, \Omega}^{2}+c\left(1 / \varepsilon_{5}\right)|\tilde{\theta}|_{2, \Omega}^{2}|h|_{3, \Omega}^{2}, \\
& J_{9} \leq \varepsilon_{6}\left|\nabla \theta^{\prime}\right|_{2, \Omega}^{2}+c\left(1 / \varepsilon_{6}\right)|v|_{6, \Omega}^{2}|\tilde{\theta}|_{3, \Omega}^{2}
\end{aligned}
$$


Using the above estimates in (3.8) yields

$$
\begin{aligned}
\frac{d}{d t}\left|\theta^{\prime}\right|_{2, \Omega}^{2}+\nu_{\varkappa}\left|\nabla \theta^{\prime}\right|_{2, \Omega}^{2} & \leq c\left(\left|\theta^{\prime}\right|_{2, \Omega}^{2}|h|_{3, \Omega}^{2}+|K|_{3, \Omega}^{2}|\chi|_{2, \Omega}^{2}\right. \\
& +|K|_{3, \Omega}^{2}\left|w_{, x^{\prime}}\right|_{2, \Omega}^{2}+|h|_{3, \Omega}^{2}\left|\mu_{, x^{\prime}}\right|_{2, \Omega}^{2}+|\tilde{\theta}|_{2, \Omega}^{2}|h|_{3, \Omega}^{2} \\
& \left.+|v|_{6, \Omega}^{2}|\tilde{\theta}|_{3, \Omega}^{2}\right)+\int_{\Omega} H \cdot \nabla \chi \cdot \theta^{\prime} d x
\end{aligned}
$$

Adding (3.7) and (3.9), using that

$$
\begin{aligned}
\int_{\Omega} H \cdot \nabla \theta \chi^{\prime} d x+\int_{\Omega} H \cdot \nabla \chi \theta^{\prime} d x & =\int_{\Omega}\left(H \cdot \nabla \tilde{\theta} \chi^{\prime}+H \cdot \nabla \tilde{\chi} \theta^{\prime}\right) d x \\
& =-\int_{\Omega}\left(H \cdot \nabla \chi^{\prime} \tilde{\theta}+H \cdot \nabla \theta^{\prime} \tilde{\chi}\right) d x \equiv I,
\end{aligned}
$$

and that

$$
|I| \leq \bar{\varepsilon}\left(\left|\nabla \chi^{\prime}\right|_{2, \Omega}^{2}+\left|\nabla \theta^{\prime}\right|_{2, \Omega}^{2}\right)+c(1 / \bar{\varepsilon})|H|_{6, \Omega}^{2}\left(|\tilde{\theta}|_{3, \Omega}^{2}+|\tilde{\chi}|_{3, \Omega}^{2}\right),
$$

and integrating the result with respect to time we obtain

$$
\begin{aligned}
\left\|\eta^{\prime}\right\|_{V_{2}^{0}\left(\Omega^{t}\right)}^{2} & \leq c\left(\|h\|_{L_{\infty}\left(0, t ; L_{3}(\Omega)\right.}^{2}+\|K\|_{L_{\infty}\left(0, t ; L_{3}(\Omega)\right)}^{2}\right. \\
& +\|\tilde{\chi}\|_{L_{2}\left(\Omega^{t}\right)}^{2}\|h\|_{L_{\infty}\left(0, t ; L_{3}(\Omega)\right)}^{2}+\|\tilde{\theta}\|_{L_{2}\left(\Omega^{t}\right)}^{2}\|h\|_{L_{\infty}\left(0, t ; L_{3}(\Omega)\right)}^{2} \\
& \left.+\|\tilde{\chi}\|_{L_{\infty}\left(0, t ; L_{3}(\Omega)\right)}^{2}+\|\tilde{\chi}\|_{L_{2}\left(0, t ; L_{4}(\Omega)\right)}^{2}+\|\tilde{\theta}\|_{L_{\infty}\left(0, t ; L_{3}(\Omega)\right)}^{2}\right) \\
& +\|\eta(0)\|_{L_{2}(\Omega)}^{2} .
\end{aligned}
$$

Next, we will use the relations (see [7])

$$
\begin{aligned}
& \int_{0}^{t}|\tilde{\chi}|_{4, \Omega}^{2} d t^{\prime} \leq c \int_{0}^{t}\left|v^{\prime}\right|_{4, \Omega}^{2} d t^{\prime} \leq c \int_{0}^{t}\left\|v^{\prime}\right\|_{H^{1}(\Omega)}^{2} d t^{\prime} \leq c \zeta_{2}^{2}, \\
& \|\tilde{\chi}\|_{L_{\infty}\left(0, t ; L_{3}(\Omega)\right)} \leq c\left\|v^{\prime}\right\|_{L_{\infty}\left(0, t ; L_{3}(S)\right)}, \\
& \int_{0}^{t}\|\tilde{\chi}\|_{L_{2}(\Omega)}^{2} d t^{\prime} \leq c \int_{0}^{t}\|v\|_{W_{2}^{1}(\Omega)}^{2} d t^{\prime} \leq c \zeta_{2}^{2}, \\
& \|\tilde{\theta}\|_{L_{\infty}\left(0, t ; L_{3}(\Omega)\right)} \leq c\left\|H^{\prime}\right\|_{L_{\infty}\left(0, t ; L_{3}(S)\right)}, \\
& \int_{0}^{t}\|\tilde{\theta}\|_{L_{2}(\Omega)}^{2} \leq c \int_{0}^{t}\|H\|_{W_{2}^{1}(\Omega)} d t^{\prime} \leq c \zeta_{2}^{2},
\end{aligned}
$$

and the transformations $\chi^{\prime}=\chi-\tilde{\chi}, \theta^{\prime}=\theta-\tilde{\theta}$ to obtain the inequality

$$
\begin{aligned}
\|\eta\|_{V_{2}^{0}\left(\Omega^{t}\right)}^{2} \leq & c \zeta_{2}^{2}\left(|h|_{L_{\infty}\left(0, t ; L_{3}(\Omega)\right)}^{2}+|K|_{L_{\infty}\left(0, t ; L_{3}(\Omega)\right)}^{2}\right) \\
& +c \zeta_{1}^{2}\left\|A^{\prime}\right\|_{L_{\infty}\left(0, t ; L_{3}(S)\right)}+c|\eta(0)|_{2, \Omega}^{2} \\
& +c\left\|A^{\prime}\right\|_{L_{\infty}\left(0, t ; H^{1 / 2+\varepsilon}(\Omega)\right)}^{2}+c\left\|A^{\prime}\right\|_{W_{2}^{1,1 / 2}\left(\Omega^{t}\right)}
\end{aligned}
$$


where we have used

$$
\begin{aligned}
\|\tilde{\chi}\|_{V_{2}^{0}\left(\Omega^{t}\right)}^{2} & \leq\|\tilde{\chi}\|_{L_{\infty}\left(0, t ; L_{2}(\Omega)\right)}^{2}+\int_{0}^{t}\|\tilde{\chi}\|_{H^{1}(\Omega)}^{2} d t^{\prime} \\
& \leq c\left\|v^{\prime}\right\|_{L_{\infty}\left(0, t ; H^{1 / 2+\varepsilon}(\Omega)\right)}^{2}+c\left\|v^{\prime}\right\|_{W_{2}^{1,1 / 2}\left(\Omega^{t}\right)}^{2}, \\
\|\tilde{\theta}\|_{V_{2}^{0}\left(\Omega^{t}\right)} & \leq c\left\|H^{\prime}\right\|_{L_{\infty}\left(0, t ; H^{1 / 2+\varepsilon}(\Omega)\right)}^{2}+c\left\|H^{\prime}\right\|_{W_{2}^{1,1 / 2}\left(\Omega^{t}\right)}^{2}
\end{aligned}
$$

Therefore we obtain from (3.11) the estimate

$$
\begin{aligned}
\|\eta\|_{V_{2}^{0}\left(\Omega^{t}\right)}^{2} \leq & c \zeta_{2}^{2}\|h, K\|_{L_{\infty}\left(0, t ; L_{3}(\Omega)\right)}^{2}+c\left(\zeta_{1}^{2}\left\|A^{\prime}\right\|_{L_{\infty}\left(0, t ; L_{3}(S)\right)}^{2}\right. \\
& \left.+\left\|A^{\prime}\right\|_{L_{\infty}\left(0, t ; H^{1 / 2+\varepsilon}(\Omega)\right)}^{2}\right)+c\left\|A^{\prime}\right\|_{W_{2}^{1,1 / 2}\left(\Omega^{t}\right)}^{2}+|\eta(0)|_{2, \Omega}^{2} .
\end{aligned}
$$

This concludes the proof of the lemma.

Let us consider the following problems:

$$
\begin{array}{ll}
v_{1, x_{2}}-v_{2, x_{1}}=\chi & \text { in } \Omega^{\prime}, \\
v_{1, x_{1}}+v_{2, x_{2}}=-h_{3} & \text { in } \Omega^{\prime}, \\
v^{\prime} \cdot \bar{n}^{\prime}=0 & \text { on } S_{1}^{\prime}, \\
H_{1, x_{2}}-H_{2, x_{1}}=\theta & \text { in } \Omega^{\prime}, \\
H_{1, x_{1}}+H_{2, x_{2}}=-K_{3} & \text { in } \Omega^{\prime}, \\
H^{\prime} \cdot \bar{n}^{\prime}=0 & \text { on } S_{1}^{\prime},
\end{array}
$$

where $\Omega^{\prime}=\Omega \cap\left\{\right.$ plane $x_{3}=$ const $\left.\in(-a, a)\right\}, S_{1}^{\prime}=S_{1} \cap$ plane $x_{3}=$ const $\epsilon$ $(-a, a)\}$, and $x_{3}, t$ are treated as parameters.

Lemma 3.2. Assume that $h(0), K(0), \eta(0) \in L_{2}(\Omega), A^{\prime}=\left(v^{\prime}, H^{\prime}\right) \in$ $L_{2}\left(\Omega ; H^{1 / 2}(0, T)\right), h, K \in L_{\infty}\left(0, T ; L_{3}(\Omega)\right)$. Then solutions of (3.13) and (3.14) satisfy

$$
\begin{aligned}
\left\|A^{\prime}\right\|_{V_{2}^{1}\left(\Omega^{t}\right)}^{2} \leq & c\left\|A^{\prime}\right\|_{L_{2}\left(\Omega ; H^{1 / 2}(0, t)\right)}^{2}+c \zeta_{2}^{2}\|h, K\|_{L_{\infty}\left(0, t ; L_{3}(\Omega)\right)}^{2} \\
& +c\left(|h(0), K(0)|_{2, \Omega}^{2}+|\eta(0)|_{2, \Omega}^{2}\right)+c\left(\zeta_{1}^{2}+\zeta_{2}^{2}\right), \quad t \leq T .
\end{aligned}
$$

Proof. For solutions to problems (3.13) and (3.14) we have

$$
\left\|A^{\prime}\right\|_{V_{2}^{1}\left(\Omega^{t}\right)} \leq c\left(\|\eta\|_{V_{2}^{0}\left(\Omega^{t}\right)}+\left\|h_{3}, K_{3}\right\|_{V_{2}^{0}\left(\Omega^{t}\right)}\right),
$$

where $\eta=(\chi, \theta)$ and $\|\eta\|_{V_{2}^{0}\left(\Omega^{t}\right)}$ is estimated by (3.12) and $\left\|h_{3}, K_{3}\right\|_{V_{2}^{0}\left(\Omega^{t}\right)}$ by (2.19).

We use these interpolation inequalities in the r.h.s. of (3.12):

$$
\begin{aligned}
& \left\|A^{\prime}\right\|_{L_{\infty}\left(0, t ; H^{1 / 2+\varepsilon}(\Omega)\right)} \leq \varepsilon\left\|A^{\prime}\right\|_{L_{\infty}\left(0, t ; H^{1}(\Omega)\right)}+c(1 / \varepsilon) \zeta_{1}, \\
& \left\|A^{\prime}\right\|_{L_{2}\left(0, t ; H^{1}(\Omega)\right)} \leq \varepsilon\left\|A^{\prime}\right\|_{L_{2}\left(0, t ; H^{2}(\Omega)\right)}+c(1 / \varepsilon) \zeta_{2} .
\end{aligned}
$$

Then

$$
\left\|A^{\prime}\right\|_{L_{\infty}\left(0, t ; L_{3}(S)\right)} \leq \varepsilon\left\|A^{\prime}\right\|_{L_{\infty}\left(0, t ; H^{1}(\Omega)\right)}+c(1 / \varepsilon) \zeta_{1} .
$$


Assuming that $\varepsilon$ is sufficiently small we obtain from (3.16)-(3.17) inequality (3.15). This concludes the proof.

To increase regularity of solutions we consider problems (1.1) and (1.2) in the form

$$
\begin{array}{ll}
v_{, t}-\operatorname{div} \mathbb{T}(v, p)=-v^{\prime} \cdot \nabla v-w h+H^{\prime} \cdot \nabla H+\mu K & \\
\quad-H_{1} \cdot \nabla H_{1}-H_{2} \cdot \nabla H_{2}-\mu \cdot \nabla \mu & \text { in } \Omega^{T}, \\
\operatorname{div} v=0 & \text { in } \Omega^{T}, \\
\bar{n} \cdot \mathbb{T}(v, p) \cdot \bar{\tau}_{\alpha}=0, \quad \alpha=1,2, & \text { on } S^{T}, \\
v \cdot \bar{n}=0 & \text { on } S^{T}, \\
\left.v\right|_{t=0}=v(0), & \text { in } \Omega,
\end{array}
$$

$H_{t}-\nu_{\varkappa} \operatorname{div} \mathbb{D}(H)=-v^{\prime} \cdot \nabla H-w K+H^{\prime} \cdot \nabla v+\mu h \quad$ in $\Omega^{T}$,

$$
\begin{array}{ll}
H \cdot \bar{n}=0, \quad \bar{n} \cdot \mathbb{D}(H) \cdot \bar{\tau}_{\alpha}=0, \quad \alpha=1,2, & \text { on } S^{T}, \\
\left.H\right|_{t=0}=H(0) & \text { in } \Omega .
\end{array}
$$

Lemma 3.3. Assume that $v(0), H(0) \in H^{1}(\Omega)$. Let

$$
\begin{aligned}
& b_{1}=\zeta_{1}+\zeta_{2}+|h(0), K(0)|_{2, \Omega}+|\eta(0)|_{2, \Omega}, \\
& b_{2}=\|h, K\|_{L_{\infty}\left(0, t ; L_{3}(\Omega)\right)}+\|h, K\|_{L_{10 / 3}\left(\Omega^{t}\right)} .
\end{aligned}
$$

Then solutions of (3.18) and (3.19) satisfy

$$
\|A\|_{W_{2}^{2,1}\left(\Omega^{t}\right)}+\|\nabla p\|_{L_{2}\left(\Omega^{t}\right)} \leq c\left(\zeta_{2} b_{2}+b_{1}\right)^{2}+\|A(0)\|_{H^{1}(\Omega)} .
$$

Proof. We have the following estimates for the r.h.s. of (3.18) and (3.19):

$$
\begin{aligned}
& \left|v^{\prime} \cdot \nabla v\right|_{5 / 3, \Omega^{t}} \leq \zeta_{2}\left|v^{\prime}\right|_{10, \Omega^{t}} \leq c \zeta_{2}\left\|v^{\prime}\right\|_{V_{2}^{1}\left(\Omega^{t}\right)}, \\
& \left|v^{\prime} \cdot \nabla H\right|_{5 / 3, \Omega^{t}} \leq c \zeta_{2}\left\|v^{\prime}\right\|_{V_{2}^{1}\left(\Omega^{t}\right)},
\end{aligned}
$$$$
|w h|_{5 / 3, \Omega^{t}}+|w K|_{5 / 3, \Omega^{t}}+|\mu K|_{5 / 3, \Omega^{t}}+|\mu h|_{5 / 3, \Omega^{t}}
$$$$
\leq|w|_{10 / 3, \Omega^{t}}|h, K|_{10 / 3, \Omega^{t}}+|\mu|_{10 / 3, \Omega^{t}}\left(|h|_{10 / 3, \Omega^{t}}+|K|_{10 / 3, \Omega^{t}}\right) \leq \zeta_{2}|h, K|_{10 / 3, \Omega^{t}},
$$
$|\mu \cdot \nabla \mu|_{5 / 3, \Omega^{t}} \leq|\mu|_{5, \Omega^{t}}|\nabla \mu|_{5 / 2, \Omega^{t}}$.

Using the imbeddings $W_{5 / 3}^{2,1}\left(\Omega^{t}\right) \hookrightarrow L_{5}\left(\Omega^{t}\right)$ and $W_{5 / 3}^{2,1}\left(\Omega^{t}\right) \hookrightarrow W_{5 / 2}^{1,0}\left(\Omega^{t}\right)$, we have $|\mu \cdot \nabla \mu|_{5 / 3, \Omega^{t}} \leq c\|\mu\|_{W_{5 / 3}^{2,1}\left(\Omega^{t}\right)^{2}}^{2}$. This implies

$$
\begin{aligned}
\|A\|_{W_{5 / 3}^{2,1}\left(\Omega^{t}\right)} \leq & c \zeta_{2}\left(\left\|A^{\prime}\right\|_{V_{2}^{1}\left(\Omega^{t}\right)}+|h|_{10 / 3, \Omega^{t}}+|K|_{10 / 3, \Omega^{t}}\right. \\
& \left.+\|A(0)\|_{W_{5 / 3}^{4 / 5}(\Omega)}+c|\mu|_{W_{5 / 3}^{2,1}\left(\Omega^{t}\right)}^{2}\right) .
\end{aligned}
$$

Now we need to estimate $\mu$ in $W_{5 / 3}^{2,1}\left(\Omega^{t}\right)$. To this end we reformulate $(2.22)_{1}$ 
in the following way:

$$
\mu_{, t}-\nu_{\varkappa} \Delta \mu=H^{\prime} \cdot \nabla w+\mu h_{3}-v^{\prime} \cdot \nabla \mu-w K_{3} .
$$

From (3.22) we have

$$
\begin{aligned}
\|\mu\|_{W_{5 / 3}^{2,1}\left(\Omega^{t}\right)} \leq & c\left(\left|H^{\prime} \cdot \nabla w\right|_{5 / 3, \Omega^{t}}+\left|v^{\prime} \cdot \nabla \mu\right|_{5 / 3, \Omega^{t}}+\left|\mu h_{3}\right|_{5 / 3, \Omega^{t}}\right. \\
& \left.+\left|w K_{3}\right|_{5 / 3, \Omega^{t}}+\|\mu(0)\|_{W_{5 / 3}^{4 / 5}(\Omega)}\right) .
\end{aligned}
$$

Using similar estimates to the r.h.s. of (3.23) as above we have

$$
\|\mu\|_{W_{5 / 3}^{2,1}\left(\Omega^{t}\right)}^{2} \leq c \zeta_{2}^{2}\left\|A^{\prime}\right\|_{V_{2}^{1}\left(\Omega^{t}\right)}^{2}+c \zeta_{2}^{2}|h, K|_{10 / 3, \Omega^{t}}^{2}
$$

Using (3.24) in (3.21) we obtain

$$
\begin{aligned}
\|A\|_{W_{5 / 3}^{2,1}\left(\Omega^{t}\right)} \leq & c \zeta_{2}^{2}\left(\left\|A^{\prime}\right\|_{V_{2}^{1}\left(\Omega^{t}\right)}+|h, K|_{10 / 3, \Omega^{t}}+\|A(0)\|_{W_{5 / 3}^{4 / 5}(\Omega)}\right) \\
& +c \zeta_{2}^{2}\left(\left\|A^{\prime}\right\|_{V_{2}^{1}\left(\Omega^{t}\right)}^{2}+|h, K|_{10 / 3, \Omega^{t}}^{2}\right) .
\end{aligned}
$$

Using Lemma 3.3 in (3.25) we obtain

$$
\|A\|_{W_{5 / 3}^{2,1}\left(\Omega^{t}\right)}+\left\|A^{\prime}\right\|_{V_{2}^{1}\left(\Omega^{t}\right)} \leq c\left(\zeta_{2} b_{2}+b_{1}\right)^{2} .
$$

Since

$$
\begin{aligned}
\left|v^{\prime} \cdot \nabla v\right|_{2, \Omega^{t}} & \leq\left|v^{\prime}\right|_{10, \Omega^{t}}|\nabla v|_{5 / 2\left(\Omega^{t}\right)} \leq\left\|v^{\prime}\right\|_{V_{2}^{1}\left(\Omega^{T}\right)}\|v\|_{W_{5 / 3}^{2,1}\left(\Omega^{t}\right)} \leq c\left(\zeta_{2} b_{2}+b_{1}\right)^{2}, \\
|w h|_{2, \Omega^{t}} & \leq\|v\|_{W_{5 / 3}^{2,1}\left(\Omega^{t}\right)}|h|_{10 / 3, \Omega^{t}} \leq c\left(\zeta_{2} b_{2}+b_{1}\right) b_{2},
\end{aligned}
$$

we obtain the same estimates for the other terms from the r.h.s. of (3.18) and (3.19).

In view of the above estimates we obtain the inequality (3.20) for solutions to problems (3.18) and (3.19). This concludes the proof.

Let us consider problems (2.17) and (2.18).

LEMma 3.4. Assume that $A \in W_{2}^{2,1}\left(\Omega^{T}\right), h, k \in L_{2}\left(\Omega^{T}\right), h(0), K(0) \in$ $W_{\sigma}^{2-2 / \sigma}(\Omega), \sigma<10$. Then solutions of problems (2.17) and (2.18) satisfy

$$
\begin{aligned}
\|h, K\|_{W_{\sigma}^{2,1}\left(\Omega^{t}\right)}+\|\nabla q\|_{L_{\sigma}\left(\Omega^{t}\right)} \leq & \phi\left(\|A\|_{W_{2}^{2,1}\left(\Omega^{t}\right)}\right)\left(|h|_{2, \Omega^{t}}+|K|_{2, \Omega^{t}}\right) \\
& +c\|h(0), K(0)\|_{W_{\sigma}^{2-2 / \sigma}(\Omega)}
\end{aligned}
$$

for all $t \leq T$, and $\phi(a)=c a^{4}$.

Proof. To estimate solutions of (2.17) and (2.18) we follow [8]. We have

$$
|v \cdot \nabla h|_{\sigma, \Omega^{t}} \leq|v|_{10, \Omega^{t}}\left(\varepsilon^{1-\varkappa}\|h\|_{W_{\sigma}^{2,1}}+c \varepsilon^{-\varkappa}|h|_{2, \Omega^{t}}\right)=I_{1}
$$

where $\varkappa=3 / 4$ because $\sigma \lambda_{1}=10[8]$. Hence

$$
I_{1} \leq \varepsilon^{1 / 4}\|h\|_{W_{\sigma}^{2,1}\left(\Omega^{t}\right)}+c \varepsilon^{-3 / 4}|v|_{10, \Omega^{t}}^{4}|h|_{2, \Omega^{t}} .
$$


Likewise

$$
\begin{aligned}
|h \cdot \nabla v|_{\sigma, \Omega^{t}} & \leq \varepsilon^{1 / 4}\|h\|_{W_{\sigma}^{2,1}\left(\Omega^{t}\right)}+c \varepsilon^{-3 / 4}|\nabla v|_{10 / 3, \Omega^{t}}^{4}|h|_{2, \Omega^{t}}, \\
|K \cdot \nabla v|_{\sigma, \Omega^{t}} & \leq \varepsilon^{1 / 4}\|K\|_{W_{\sigma}^{2,1}\left(\Omega^{t}\right)}+c \varepsilon^{-3 / 4}|\nabla v|_{10 / 3, \Omega^{t}}^{4}|K|_{2, \Omega^{t}}, \\
|v \cdot \nabla K|_{\sigma, \Omega^{t}} & \leq \varepsilon^{1 / 4}\|K\|_{W_{\sigma}^{2,1}\left(\Omega^{t}\right)}+c \varepsilon^{-3 / 4}|v|_{10, \Omega^{t}}^{4}|K|_{2, \Omega^{t}}, \\
|H \cdot \nabla h|_{\sigma, \Omega^{t}} & \leq \varepsilon^{1 / 4}\|h\|_{W_{\sigma}^{2,1}\left(\Omega^{t}\right)}+c \varepsilon^{-3 / 4}|H|_{10, \Omega^{t}}^{4}|h|_{2, \Omega^{t}}, \\
|h \cdot \nabla H|_{\sigma, \Omega^{t}} & \leq \varepsilon^{1 / 4}\|h\|_{W_{\sigma}^{2,1}\left(\Omega^{t}\right)}+c \varepsilon^{-3 / 4}|\nabla H|_{10 / 3, \Omega^{t}}^{4}|h|_{2, \Omega^{t}} .
\end{aligned}
$$

Adding all estimates we get

$$
\begin{aligned}
\|h, K\|_{W_{\sigma}^{2,1}\left(\Omega^{t}\right)}+\|\nabla q\|_{L_{\sigma}\left(\Omega^{t}\right)} \leq & \phi\left(\|A\|_{W_{2}^{2,1}\left(\Omega^{t}\right)}\right)|h, K|_{2, \Omega^{t}} \\
& +c\|h(0), K(0)\|_{W_{\sigma}^{2-2 / \sigma}\left(\Omega^{t}\right)}
\end{aligned}
$$

for $\phi(a)=c a^{4}$. This concludes the proof.

Lemma 3.5. With the assumptions of Lemma 3.4, for $\sigma>5 / 3$ there exists a sufficiently large constant $L$ such that

$$
\|h, K\|_{W_{\sigma}^{2,1}\left(\Omega^{t}\right)}+\|\nabla q\|_{L_{\sigma}\left(\Omega^{t}\right)} \leq L .
$$

Proof. Since $b_{2} \leq c\|h, K\|_{W_{\sigma}^{2,1}\left(\Omega^{t}\right)}$, for $\sigma>5 / 3$, we obtain

$$
\begin{aligned}
\|h, K\|_{W_{\sigma}^{2,1}\left(\Omega^{t}\right)}+|\nabla q|_{0, \Omega^{t}} \leq & \phi\left(\|h\|_{W_{\sigma}^{2,1}},\|K\|_{W_{\sigma}^{2,1}}, b_{1}\right) \\
& \cdot|h(0), K(0)|_{2, \Omega}+c\|h(0), K(0)\|_{W_{\sigma}^{2-2 / \sigma}(\Omega)} .
\end{aligned}
$$

For $\delta$ sufficiently small such that $|h(0), K(0)|_{2, \Omega^{t}}<\delta$ there exists $L$ such that

$$
\phi\left(L, b_{1}\right) \delta+c\|h(0), K(0)\|_{W_{\sigma}^{2-2 / \sigma}(\Omega)} \leq L \quad \text { for } L>b_{3}
$$

where $b_{3}=\|h(0), K(0)\|_{W_{\sigma}^{2-2 / \sigma}(\Omega)}$. Hence the estimate (3.28) holds. This concludes the proof.

4. Existence. To prove the existence of solutions we consider the problems

$$
\begin{aligned}
& h_{t}-\operatorname{div} \mathbb{T}(h, q)=-\lambda[v(\tilde{h}, \tilde{K}) \cdot \nabla \tilde{h}+\tilde{h} \cdot \nabla v(\tilde{h}, \tilde{K}) \\
& +\tilde{K}_{i} \cdot \nabla H_{i}(\tilde{K}, \tilde{h})+H_{i}(\tilde{K}, \tilde{h}) \cdot \nabla \tilde{K}_{i} \\
& -\tilde{K} \cdot \nabla H(\tilde{K}, \tilde{h})-H(\tilde{K}, \tilde{h}) \cdot \nabla \tilde{K}] \quad \text { in } \Omega^{T}, \\
& \operatorname{div} h=0 \quad \text { in } \Omega^{T}, \\
& h \cdot \bar{n}=0, \quad \bar{n} \cdot \mathbb{D}(h) \cdot \bar{\tau}_{\alpha}=0, \quad \alpha=1,2, \quad \text { on } S_{1}^{T}, \\
& h_{i}=0, \quad i=1,2, \quad h_{3, x_{3}}=0 \quad \text { on } S_{2}^{T}, \\
& h_{i}=0, \quad i=1,2, \quad h_{3, x_{3}}=0,\left.\quad h\right|_{t=0}=h(0) \quad \text { in } \Omega,
\end{aligned}
$$


and

$$
\begin{aligned}
& K_{t}-\nu_{\varkappa} \Delta K=\lambda[\tilde{K} \cdot \nabla v(\tilde{h}, \tilde{K})+H(\tilde{K}, \tilde{h}) \cdot \nabla \tilde{h} \\
& -\tilde{h} \cdot \nabla H(\tilde{K}, \tilde{h})-v(\tilde{h}, \tilde{K}) \cdot \nabla \tilde{K}] \quad \text { in } \Omega^{T}, \\
& K \cdot \bar{n}=0, \quad \bar{n} \cdot \mathbb{D}(K) \cdot \bar{\tau}_{\alpha}=0, \quad \alpha=1,2, \quad \text { on } S_{1}^{T}, \\
& K_{j}=0, j=1,2, K_{3, x_{3}}=0 \quad \text { on } S_{2}^{T} \text {, } \\
& \left.K\right|_{t=0}=K(0) \quad \text { in } \Omega \text {, }
\end{aligned}
$$

where $\lambda \in[0,1]$. The functions $v=v(\tilde{h}, \tilde{K}), H=H(\tilde{v}, \tilde{K})$ are constructed in Lemmas 2.6, 2.7, 3.1, 3.2 and 3.3. Let $\mathcal{M}\left(\Omega^{T}\right)=\left\{(h, K):\|h, K\|_{L_{\infty}\left(0, T ; W_{\eta}^{1}(\Omega)\right)}\right.$ $<\infty\}$.

Problems (4.1), (4.2) yield a mapping

$$
\Phi: \mathcal{M}\left(\Omega^{T}\right) \rightarrow W_{\sigma}^{2,1}\left(\Omega^{T}\right) \times W_{\sigma}^{2,1}\left(\Omega^{T}\right) \hookrightarrow \mathcal{M}\left(\Omega^{T}\right)
$$

where the last imbedding and so the mapping $\Phi$ is compact for $5 / \sigma-3 / \eta<1$, and $\eta>4$.

First we show the continuity of the mapping $\Phi$.

LEMMA 4.1. The mapping $\Phi$ is uniformly continuous and compact in the product $\mathcal{M}\left(\Omega^{T}\right) \times[0,1]$ where $\mathcal{M}\left(\Omega^{T}\right)$ is defined above and $20 / 7<\sigma \leq 10 / 3$, $\eta>4$.

Proof. Uniform continuity with respect to $\lambda \in[0,1]$ is evident. Therefore we examine the uniform continuity with respect to elements of $\mathcal{M}\left(\Omega^{T}\right)$ for any $\lambda \in[0,1]$. Since dependence on $\lambda$ is very simple we omit $\lambda$ in the considerations below because it does not affect the proof. The imbeddings and interpolation inequalities used in this section follow from [1, Ch. 3, Sect. 10].

To have $\Phi$ compact we need the compactness of the imbedding

$$
W_{\sigma}^{2,1}\left(\Omega^{T}\right) \hookrightarrow L_{\infty}\left(0, T ; W_{\eta}^{1}(\Omega)\right), \quad \text { so } \quad \frac{5}{\sigma}-\frac{3}{\eta}<1, \quad \sigma<\eta .
$$

Let $\left(\tilde{h}_{s}, \tilde{K}_{s}\right) \in \mathcal{M}\left(\Omega^{T}\right) \times \mathcal{M}\left(\Omega^{T}\right), s=1,2$, be two elements. We consider the following problems for $s=1,2$ :

$$
\begin{aligned}
& h_{s, t}-\operatorname{div} \mathbb{T}\left(h_{s}, q_{s}\right)=-v_{s} \cdot \nabla \tilde{h}_{s}-\tilde{h}_{s} \cdot \nabla v_{s} \\
& -\tilde{K}_{s i} \cdot \nabla H_{s i}-H_{s i} \cdot \nabla \tilde{K}_{s i}+\tilde{K}_{s} \cdot \nabla H_{s}+H_{s} \cdot \nabla \tilde{K}_{s} \quad \text { in } \Omega^{T}, \\
& \operatorname{div} h_{s}=0 \quad \text { in } \Omega^{T}, \\
& h_{s} \cdot \bar{n}=0, \quad \bar{n} \cdot \mathbb{D}\left(h_{s}\right) \cdot \bar{\tau}_{\alpha}=0, \quad \alpha=1,2, \quad \text { on } S_{1}^{T}, \\
& h_{s i}=0, \quad i=1,2, \quad h_{s 3, x_{3}}=0 \quad \text { on } S_{2}^{T} \text {, } \\
& \left.h_{s}\right|_{t=0}=h_{s}(0) \quad \text { in } \Omega \text {, }
\end{aligned}
$$




$$
\begin{array}{lr}
\begin{array}{lr}
K_{s, t}-\nu_{\varkappa} \Delta K_{s}=-\tilde{h}_{s} \cdot \nabla H_{s}-v_{s} \cdot \nabla \tilde{K}_{s}+\tilde{K}_{s} \cdot \nabla v \\
\quad+H_{s} \cdot \nabla \tilde{h}_{s} & \text { in } \Omega^{T}, \\
\operatorname{div} K_{s}=0 & \text { in } \Omega^{T}, \\
\bar{n} \cdot K_{s}=0, \quad \bar{n} \cdot \mathbb{D}\left(K_{s}\right) \cdot \bar{\tau}_{\alpha}=0, \quad \alpha=1,2, & \text { on } S^{T}, \\
\left.K_{s}\right|_{t=0}=K(0) & \text { in } \Omega, \\
\chi_{s, t}+v_{s} \cdot \nabla \chi_{s}-\tilde{h}_{s 3} \chi_{s}+w_{s, x_{1}} \tilde{h}_{s 2}-\tilde{h}_{s 1} w_{s, x_{2}} & \\
\quad+\tilde{K}_{s 3} \theta_{s}-\tilde{K}_{s 2} \mu_{s, x_{1}}+\mu_{s, x_{2}} \tilde{K}_{s 1}-H_{s} \cdot \nabla \theta_{s} & \\
\quad-\nu \Delta \chi_{s}=0 & \text { in } \Omega^{T}, \\
\chi_{s}=\chi_{s *} & \text { on } S_{1}^{T}, \\
\chi_{s, x_{3}}=0 & \text { on } S_{2}^{T}, \\
\left.\chi_{s}\right|_{t=0}=\chi(0) & \text { in } \Omega,
\end{array}
\end{array}
$$

and

$$
\begin{aligned}
& \theta_{s, t}+v_{s} \cdot \nabla \theta_{s}-\tilde{h}_{s 3} \theta_{s}-\tilde{K}_{s 3} \chi_{s}+w_{s, x_{1}} \tilde{K}_{s 2} \\
& -w_{s, x_{2}} \tilde{K}_{s 1}+\mu_{s, x_{1}} \tilde{h}_{s 2}-\mu_{s, x_{2}} \tilde{h}_{s 1}-H_{s} \cdot \nabla \chi_{s} \\
& -\nu_{\varkappa} \Delta \theta_{s}=0 \\
& \text { in } \Omega^{T} \text {, } \\
& \theta_{s}=\theta_{s *} \\
& \text { on } S_{1}^{T} \\
& \theta_{s}=0 \\
& \text { on } S_{2}^{T} \text {, } \\
& \left.\theta_{s}\right|_{t=0}=\theta(0) \\
& \text { in } \Omega \text {. }
\end{aligned}
$$

Next we consider the elliptic problems

and

$$
\begin{array}{ll}
v_{s 1, x_{2}}-v_{s 2, x_{1}}=\chi_{s} & \text { in } \Omega^{\prime}, \\
v_{s 1, x_{1}}+v_{s 2, x_{2}}=-\tilde{h}_{s 3} & \text { in } \Omega^{\prime}, \\
v_{s^{\prime}} \cdot \bar{n}^{\prime}=0 & \text { on } S_{1}^{\prime},
\end{array}
$$

$$
\begin{array}{ll}
H_{s 1, x_{2}}-H_{s 2, x_{1}}=\theta_{s} & \text { in } \Omega^{\prime}, \\
H_{s 1, x_{1}}+H_{s 2, x_{2}}=-\tilde{K}_{s 3} & \text { in } \Omega^{\prime}, \\
H_{s}^{\prime} \cdot \bar{n}^{\prime}=0 & \text { on } S_{1}^{\prime},
\end{array}
$$

where $s=1,2$, and $\Omega^{\prime}$ and $S_{1}^{\prime}$ are the intersections of $\Omega$ and $S_{1}$ with a plane perpendicular to the $x_{3}$ axis.

Following [8] we examine the problem for $\chi$. Let us introduce the function $\tilde{\chi}_{s}$ as a solution to the problem

$$
\begin{array}{ll}
\tilde{\chi}_{s, t}-\nu \Delta \tilde{\chi}_{s}=0 & \text { in } \Omega^{T}, \\
\tilde{\chi}_{s}=\chi_{s_{*}} & \text { on } S_{1}^{T}, \\
\tilde{\chi}_{s, x_{3}}=0 & \text { on } S_{2}^{T}, \\
\left.\tilde{\chi}_{s}\right|_{t=0}=0 & \text { in } \Omega,
\end{array}
$$


where $s=1,2$. Introducing the new function

$$
\chi_{s}^{\prime}=\chi_{s}-\tilde{\chi}_{s}, \quad s=1,2,
$$

we see that it is a solution to the problem

$$
\begin{aligned}
& \chi_{s, t}^{\prime}+v_{s} \cdot \nabla \chi_{s}^{\prime}-\tilde{h}_{s 3} \chi_{s}^{\prime}-\tilde{h}_{s 1} w_{s, x_{2}}+\tilde{h}_{s 2} w_{s, x_{1}} \\
& +\tilde{K}_{s 1} \mu_{s, x_{2}}-\tilde{K}_{s 2} \mu_{s, x_{1}}+\tilde{K}_{s 3} \theta_{s}-H_{s} \cdot \nabla \theta_{s} \\
& -\nu \Delta \chi_{s}^{\prime}=\tilde{h}_{s 3} \tilde{\chi}_{s}-v_{s} \cdot \nabla \tilde{\chi}_{s} \quad \text { in } \Omega^{T}, \\
& \chi_{s}^{\prime}=0 \quad \text { on } S_{1}^{T} \text {, } \\
& \chi_{s, x_{3}}^{\prime}=0 \quad \text { on } S_{2}^{T} \text {, } \\
& \left.\chi_{s}^{\prime}\right|_{t=0}=\chi(0) \quad \text { in } \Omega .
\end{aligned}
$$

The problems for $v_{s}$ and $H_{s}$ read:

$$
\begin{aligned}
& v_{s, t}-\operatorname{div} \mathbb{T}\left(v_{s}, p_{s}\right)=v_{s}^{\prime} \cdot \nabla^{\prime} v_{s}-w_{s} \tilde{h}_{s}+H_{s}^{\prime} \cdot \nabla^{\prime} H_{s} \\
& +\mu_{s} \tilde{K}_{s}-H_{s 1} \cdot \nabla H_{s 1}-H_{s 2} \cdot \nabla H_{s 2}-\mu_{s} \cdot \nabla \mu_{s} \quad \text { in } \Omega^{T}, \\
& \operatorname{div} v_{s}=0 \quad \text { in } \Omega^{T}, \\
& \bar{n} \cdot \mathbb{T}\left(v_{s}, p_{s}\right) \cdot \bar{\tau}_{\alpha}=v_{s} \cdot \bar{n}=0, \quad i=1,2, \quad \text { on } S^{T}, \\
& \left.v_{s}\right|_{t=0}=v(0) \quad \text { in } \Omega \text {, }
\end{aligned}
$$$$
H_{s, t}-\nu_{\varkappa} \Delta H_{s}=v_{s}^{\prime} \cdot \nabla^{\prime} H_{s}-w_{s} \tilde{K}_{s}+H_{s}^{\prime} \cdot \nabla^{\prime} v_{s}+\mu_{s} \tilde{h}_{s} \quad \text { in } \Omega^{T},
$$$$
\operatorname{div} H_{s}=0 \quad \text { in } \Omega^{T},
$$$$
\bar{n} \cdot H_{s}=0, \quad \bar{n} \cdot \mathbb{D}\left(H_{s}\right) \cdot \bar{\tau}_{\alpha}=0, \quad \alpha=1,2, \quad \text { on } S^{T},
$$$$
\left.H_{s}\right|_{t=0}=H(0) \quad \text { in } \Omega .
$$

For $v_{s}$ and $H_{s}$ we have an estimate of the form (3.20),

$$
\left\|A_{s}\right\|_{W_{2}^{2,1}\left(\Omega^{T}\right)}+\left\|\nabla p_{s}\right\|_{L_{2}\left(\Omega^{T}\right)} \leq c\left(\zeta_{2} b_{2}+b_{1}\right)^{2}+c\left\|A_{s}(0)\right\|_{H^{1}(\Omega)},
$$

with

$$
\begin{aligned}
& b_{1}:=c\left(\zeta_{1}+\zeta_{2}\right)+c\left(\left|\tilde{h}_{s}(0)\right|_{2, \Omega}^{2}+\left|\tilde{K}_{s}(0)\right|_{2, \Omega}^{2}+\left|\eta_{s}(0)\right|_{2, \Omega}^{2}\right), \\
& b_{2}:=\left\|\tilde{h}_{s}\right\|_{L_{\infty}\left(0, t ; L_{3}(\Omega)\right)}+\left\|\tilde{K}_{s}\right\|_{L_{\infty}\left(0, t ; L_{3}(\Omega)\right)}+\left\|\tilde{h}_{s}\right\|_{L_{10 / 3}\left(\Omega^{T}\right)} \\
& \quad+\left\|\tilde{K}_{s}\right\|_{10 / 3\left(\Omega^{T}\right)}, \\
& \left\|A_{s}\right\|_{X} \equiv\left\|v_{s}\right\|_{X}+\left\|H_{s}\right\|_{X}, \\
& \left\|\eta_{s}\right\|_{X}=\left\|\chi_{s}\right\|_{X}+\left\|\theta_{s}\right\|_{X},
\end{aligned}
$$

where $b_{1}$ and $b_{2}$ depend on $\tilde{h}_{s}, \tilde{K}_{s}$ instead of $h_{s}, K_{s}$.

Therefore, since $\mathcal{M}\left(\Omega^{T}\right) \hookrightarrow L_{\infty}\left(0, T ; L_{3}(\Omega)\right)$ and $\mathcal{M}\left(\Omega^{T}\right) \hookrightarrow L_{10 / 3}\left(\Omega^{T}\right)$ we can replace this relation with

$$
\begin{aligned}
\left\|A_{s}\right\|_{W_{2}^{2,1}\left(\Omega^{T}\right)}+ & \left\|\nabla p_{s}\right\|_{L_{2}\left(\Omega^{T}\right)} \\
& \leq c\left[\left(\zeta_{1}\left\|\tilde{h}_{s}, \tilde{K}_{s}\right\|_{\mathcal{M}\left(\Omega^{T}\right)}+b_{1}\right)^{2}+\left\|A_{s}(0)\right\|_{H^{1}(\Omega)}\right] .
\end{aligned}
$$


For problems (4.3) and (4.4) and the functions $\left(h_{s}, K_{s}\right)$ we have

$$
\begin{aligned}
\left\|h_{s}, K_{s}\right\|_{W_{\sigma}^{2,1}\left(\Omega^{T}\right)} & +\left\|\nabla q_{s}\right\|_{L_{\sigma}\left(\Omega^{T}\right)} \leq c\left[\left\|v_{s} \cdot \nabla \tilde{h}_{s}\right\|_{L_{\sigma}\left(\Omega^{T}\right)}\right. \\
& +\left\|\tilde{h}_{s} \cdot \nabla v_{s}\right\|_{L_{\sigma}\left(\Omega^{T}\right)}+\left\|\tilde{K}_{s i} \cdot \nabla H_{s i}\right\|_{L_{\sigma}\left(\Omega^{T}\right)} \\
& +\left\|H_{s i} \cdot \nabla \tilde{K}_{s i}\right\|_{L_{\sigma}\left(\Omega^{T}\right)}+\left\|\tilde{K}_{s} \cdot \nabla H_{s}\right\|_{L_{\sigma}\left(\Omega^{T}\right)} \\
& +\left\|H_{s} \cdot \nabla \tilde{K}_{s}\right\|_{L_{\sigma}\left(\Omega^{T}\right)}+\left\|\tilde{h}_{s} \cdot \nabla H_{s}\right\|_{L_{\sigma}\left(\Omega^{T}\right)} \\
& +\left\|v_{s} \cdot \nabla \tilde{K}_{s}\right\|_{L_{\sigma}\left(\Omega^{T}\right)}+\left\|\tilde{K}_{s} \cdot \nabla v_{s}\right\|_{L_{\sigma}\left(\Omega^{T}\right)} \\
& +\left\|H_{s} \cdot \nabla \tilde{h}_{s}\right\|_{L_{\sigma}\left(\Omega^{T}\right)}+\left\|h_{s}(0), K_{s}(0)\right\|_{W_{\sigma}^{2-2 / \sigma}(\Omega)}
\end{aligned}
$$

for $\left\|h_{s}, K_{s}\right\|_{X} \equiv\left\|h_{s}\right\|_{X}+\left\|K_{s}\right\|_{X}$.

Note that we cannot apply directly the results analogous to Lemma 3.4 and instead we need to estimate the r.h.s. of (4.15) in a different way.

We split the first term on the r.h.s. of (4.15) into (see [8])

$$
I_{1} \equiv\left\|v_{s} \cdot \nabla \tilde{h}_{s}\right\|_{L_{\sigma}\left(\Omega^{t}\right)} \leq\left\|v_{s}\right\|_{L_{\sigma \lambda_{1}}\left(\Omega^{t}\right)}\left\|\nabla \tilde{h}_{s}\right\|_{L_{\sigma \lambda_{2}}\left(\Omega^{t}\right)}
$$

with $1 / \lambda_{1}+1 / \lambda_{2}=1$. We estimate $I_{1}$ under the assumptions that $W_{2}^{2,1}\left(\Omega^{t}\right)$ $\hookrightarrow L_{\sigma \lambda_{1}}\left(\Omega^{t}\right)$ and $\tilde{h}_{s} \in L_{\eta}\left(\Omega^{t}\right)$. Therefore, we have the following relations:

$$
\frac{5}{2}-\frac{5}{\sigma \lambda_{1}} \leq 2, \quad \sigma \lambda_{2} \leq \eta \text {. }
$$

Let $\sigma \lambda_{2}=\eta$. Then

$$
\frac{5}{\sigma \lambda_{1}} \geq \frac{1}{2} \Rightarrow \frac{5}{\sigma}\left(\frac{1}{\lambda_{1}}+\frac{1}{\lambda_{2}}\right)-\frac{5}{\eta} \geq \frac{1}{2} \Rightarrow \frac{5}{\sigma}-\frac{5}{\eta} \geq \frac{1}{2} .
$$

We combine this relation with the compactness condition to get

$$
\frac{1}{2}+\frac{2}{\eta} \leq \frac{5}{\sigma}-\frac{3}{\eta}<1
$$

and we deduce

$$
\eta>4, \quad \sigma>20 / 7 .
$$

The second term on the r.h.s. of (4.15) is estimated by

$$
I_{2} \equiv\left\|\tilde{h}_{s} \cdot \nabla v_{\sigma \mu_{1}}\right\|_{L_{\sigma}\left(\Omega^{t}\right)} \leq\left\|\nabla v_{s}\right\|_{L_{\sigma \mu_{1}}\left(\Omega^{t}\right)}\left\|\tilde{h}_{s}\right\|_{L_{\sigma \mu_{2}}\left(\Omega^{t}\right)}
$$

with $1 / \mu_{1}+1 / \mu_{2}=1$. Since $\tilde{h}_{s} \in L_{\infty}\left(0, t ; W_{\eta}^{1}(\Omega)\right)$ with $\eta>4$ we have $\tilde{h}_{s} \in L_{\infty}\left(0, t ; L_{\varrho}(\Omega)\right)$ with arbitrary $\varrho \leq \infty$. Therefore we set $\mu_{2}=\infty$ and $\mu_{1}=1$. Consequently, for $v_{s} \in W_{2}^{2,1}\left(\Omega^{t}\right) \hookrightarrow L_{\sigma}\left(0, t ; W_{\sigma}^{1}(\Omega)\right)$ we have the relation

Hence

$$
\frac{5}{2}-\frac{5}{\sigma} \leq 1
$$

$$
\sigma \leq 10 / 3
$$


Because $\tilde{K}_{s} \in L_{\infty}\left(0, t ; W_{\eta}^{1}(\Omega)\right), \eta>4$, similar estimates on the r.h.s. of (4.15) imply

$$
\begin{aligned}
& \left\|h_{s}, K_{s}\right\|_{W_{\sigma}^{2,1}\left(\Omega^{T}\right)}+\left\|\nabla q_{s}\right\|_{L_{\sigma}\left(\Omega^{T}\right)} \\
& \quad \leq c\left[\left\|A_{s}\right\|_{W_{2}^{2,1}\left(\Omega^{T}\right)}+\left\|\tilde{h}_{s}, \tilde{K}_{s}\right\|_{\mathcal{M}\left(\Omega^{T}\right)}+\left\|h_{s}(0), K_{s}(0)\right\|_{W_{\sigma}^{2-2 / \sigma}(\Omega)}\right] .
\end{aligned}
$$

Next, we also use the estimate on $A_{s}$, i.e. (4.14) to infer the inequality

$$
\begin{aligned}
& \left\|h_{s}, K_{s}\right\|_{W_{\sigma}^{2,1}\left(\Omega^{T}\right)}+\left\|\nabla q_{s}\right\|_{L_{\sigma}\left(\Omega^{T}\right)} \\
& \quad \leq \varphi\left(\left\|\tilde{h}_{s}, \tilde{K}_{s}\right\|_{\mathcal{M}\left(\Omega^{T}\right)}, b_{3}\right)+c\left\|h_{s}(0), K_{s}(0)\right\|_{W_{\sigma}^{2-2 / \sigma}(\Omega)},
\end{aligned}
$$

where $b_{3}:=b_{1}=\left\|v_{s}(0)\right\|_{H^{1}(\Omega)}$ and $\varphi$ is an increasing positive function.

This proves that bounded sets in $\mathcal{M}\left(\Omega^{T}\right)$ are transformed into bounded sets in $\mathcal{M}\left(\Omega^{T}\right)$.

To show continuity we formulate problems for the differences

$$
\begin{array}{ll}
K^{(r)}=K_{1}-K_{2}, & h^{(r)}=h_{1}-h_{2}, \quad q^{(r)}=q_{1}-q_{2}, \\
v^{(r)}=v_{1}-v_{2}, & H^{(r)}=H_{1}-H_{2} .
\end{array}
$$

Thus $h^{(r)}$ and $K^{(r)}$ satisfy

$$
\begin{aligned}
& h_{, t}^{(r)}-\operatorname{div} \mathbb{T}\left(h^{(r)}, q^{(r)}\right)=-v^{(r)} \cdot \nabla \tilde{h}_{1}-v_{2} \cdot \nabla \tilde{h}^{(r)} \\
& -\tilde{h}^{(r)} \cdot \nabla v_{1}-\tilde{h}_{2} \cdot \nabla v^{(r)}-\tilde{K}_{i}^{(r)} \nabla H_{1 i} \\
& -\tilde{K}_{2 i} \nabla H_{i}^{(r)}-H_{i}^{(r)} \nabla \tilde{K}_{1 i}-H_{2 i} \nabla \tilde{K}_{i}^{(r)} \\
& +\tilde{K}^{(r)} \cdot \nabla H_{1}+\tilde{K}_{2} \cdot \nabla H^{(r)}+H^{(r)} \cdot \nabla \tilde{K}_{1} \\
& +H_{2} \cdot \nabla \tilde{K}^{(r)} \quad \text { in } \Omega^{T}, \\
& \operatorname{div} h^{(r)}=0 \\
& \bar{n} \cdot \mathbb{D}\left(h^{(r)}\right) \cdot \bar{\tau}_{\alpha}=h^{(r)} \cdot \bar{n}=0 \\
& \text { in } \Omega^{T} \text {, } \\
& h_{j}^{(r)}=0, \quad j=1,2, \quad h_{3, x_{3}}^{(r)}=0 \\
& \text { on } S_{1}^{T} \text {, } \\
& \left.h^{(r)}\right|_{t=0}=0 \\
& \text { on } S_{2}^{T} \text {, } \\
& \text { in } \Omega \text {, }
\end{aligned}
$$

and

$$
\begin{array}{ll}
K_{, t}^{(r)}-\nu_{\varkappa} \Delta K^{(r)}=-\tilde{h}^{(r)} \cdot \nabla H_{1}-\tilde{h}_{2} \cdot \nabla H^{(r)} & \\
\quad-v^{(r)} \cdot \nabla \tilde{K}_{1}-v_{2} \cdot \nabla \tilde{K}^{(r)}+\tilde{K}^{(r)} \cdot \nabla v_{1} & \\
\quad+\tilde{K}_{2} \cdot \nabla v^{(r)}+H^{(r)} \cdot \nabla \tilde{h}_{1}+H_{2} \cdot \nabla \tilde{h}^{(r)} & \text { in } \Omega^{T}, \\
\operatorname{div} K^{(r)}=0 & \text { in } \Omega^{T}, \\
\bar{n} \cdot K^{(r)}=0, \quad \bar{n} \cdot \mathbb{D}\left(K^{(r)}\right) \cdot \bar{\tau}_{\alpha}, \quad \alpha=1,2, & \text { on } S_{1}^{T}, \\
K_{j}^{(r)}=0, \quad j=1,2, \quad K_{3, x_{3}}^{(r)}=0 & \text { on } S_{2}^{T}, \\
\left.K^{(r)}\right|_{t=0}=0 & \text { in } \Omega .
\end{array}
$$


For solutions of (4.19) and (4.20) we have

$$
\begin{aligned}
\| h^{(r)}, K^{(r)} & \left\|_{W_{\sigma}^{2,1}\left(\Omega^{t}\right)}+\right\| \nabla q^{(r)} \|_{L_{\sigma}\left(\Omega^{t}\right)} \\
\leq & \left.c|| v^{(r)} \cdot \nabla \tilde{h}_{1}\right|_{\sigma, \Omega^{t}}+\left|v_{2} \cdot \nabla \tilde{h}^{(r)}\right|_{\sigma, \Omega^{t}} \\
& +\left|\tilde{h}_{2} \cdot \nabla v^{(r)}\right|_{\sigma, \Omega^{t}}+\left|h^{(r)} \cdot \nabla v_{1}\right|_{\sigma, \Omega^{t}}+\left|\tilde{K}_{i}^{(r)} \nabla H_{1 i}\right|_{\sigma, \Omega^{t}} \\
& +\left|\tilde{K}_{2 i} \nabla H_{i}^{(r)}\right|_{\sigma, \Omega^{t}}+\left|H_{i}^{(r)} \nabla \tilde{K}_{1 i}\right|_{\sigma, \Omega^{t}}+\left|H_{2 i} \nabla \tilde{K}_{i}^{(r)}\right|_{\sigma, \Omega^{t}} \\
& +\left|K^{(r)} \cdot \nabla H_{1}\right|_{\sigma, \Omega^{t}}+\left|\tilde{K}_{2} \cdot \nabla H^{(r)}\right|_{\sigma, \Omega^{t}}+\left|H^{(r)} \nabla \tilde{K}_{1}\right|_{\sigma, \Omega^{t}} \\
& +\left|H_{2} \nabla \tilde{K}^{(r)}\right|_{\sigma, \Omega^{t}}+\left|\tilde{h}^{(r)} \cdot \nabla H_{1}\right|_{\sigma, \Omega^{t}}+\left|\tilde{h}_{2} \cdot \nabla H^{(r)}\right|_{\sigma, \Omega^{t}} \\
& +\left|v^{(r)} \cdot \nabla \tilde{K}_{1}\right|_{\sigma, \Omega^{t}}+\left|v_{2} \cdot \nabla \tilde{K}^{(r)}\right|_{\sigma, \Omega^{t}}+\left|\tilde{K}^{(r)} \cdot \nabla v_{1}\right|_{\sigma, \Omega^{t}} \\
& \left.+\left|\tilde{K}_{2} \cdot \nabla v^{(r)}\right|_{\sigma, \Omega^{t}}+\left|H^{(r)} \cdot \nabla \tilde{h}_{1}\right|_{\sigma, \Omega^{t}}+\left|H_{2} \cdot \nabla \tilde{h}^{(r)}\right|_{\sigma, \Omega^{t}}\right] .
\end{aligned}
$$

Applying the Hölder inequality (4.21) implies

$$
\begin{aligned}
&\left\|h^{(r)}, K^{(r)}\right\|_{W_{\sigma}^{2,1}\left(\Omega^{t}\right)}+\left|\nabla q^{(r)}\right|_{\sigma, \Omega^{t}} \\
& \leq\left.c|| v^{(r)}\right|_{\sigma \delta_{1}^{1}, \Omega^{t}}\left|\nabla \tilde{h}_{1}\right|_{\sigma \delta_{2}^{1}, \Omega^{t}} \\
&+\left|v_{2}\right|_{\sigma \delta_{1}^{2}, \Omega^{t}}\left|\nabla \tilde{h}^{(r)}\right|_{\sigma \delta_{2}^{2}, \Omega^{t}}+\left|\tilde{K}_{i}^{(r)}\right|_{\sigma \delta_{1}^{3}, \Omega^{t}}\left|\nabla H_{1 i}\right|_{\sigma \delta_{2}^{3}, \Omega^{t}} \\
&+\left|\tilde{K}_{2 i}\right|_{\sigma \delta_{1}^{4}, \Omega^{t}}\left|\nabla H_{i}^{(r)}\right|_{\sigma \delta_{2}^{4}, \Omega^{t}}+\left|H_{i}^{(r)}\right|_{\sigma \delta_{1}^{5}, \Omega^{t}}\left|\nabla \tilde{K}_{1 i}\right|_{\sigma \delta_{2}^{5}, \Omega^{t}} \\
&+\left|H_{2 i}\right|_{\sigma \delta_{1}^{6}, \Omega^{t}}\left|\nabla \tilde{K}_{i}^{(r)}\right|_{\sigma \delta_{2}^{6}, \Omega^{t}}+\left|\tilde{K}^{(r)}\right|_{\sigma \delta_{1}^{7}, \Omega^{t}}\left|\nabla H_{1}\right|_{\sigma \delta_{2}^{7}, \Omega^{t}} \\
&+\left|\tilde{K}_{2}\right|_{\sigma \delta_{1}^{8}, \Omega^{t}}\left|\nabla H^{(r)}\right|_{\sigma \delta_{2}^{8}, \Omega^{t}}+\left|H^{(r)}\right|_{\sigma \delta_{1}^{9}, \Omega^{t}}\left|\nabla \tilde{K}_{1}\right|_{\sigma \delta_{2}^{9}, \Omega^{t}} \\
&+\left|H_{2}\right|_{\sigma \delta_{1}^{10}, \Omega^{t}}\left|\nabla \tilde{K}^{(r)}\right|_{\sigma \delta_{2}^{10}, \Omega^{t}}+\left|\tilde{h}^{(r)}\right|_{\sigma \delta_{1}^{11}, \Omega^{t}}\left|\nabla H_{1}\right|_{\sigma \delta_{2}^{11}, \Omega^{t}} \\
&+\left|\tilde{h}_{2}\right|_{\sigma \delta_{1}^{12}, \Omega^{t}}\left|\nabla H^{(r)}\right|_{\sigma \delta_{2}^{12}, \Omega^{t}}+\left|v^{(r)}\right|_{\sigma \delta_{1}^{13}, \Omega^{t}}\left|\nabla \tilde{K}_{1}\right|_{\sigma \delta_{2}^{13}, \Omega^{t}} \\
&+\left|v_{2}\right|_{\sigma \delta_{1}^{14}, \Omega^{t}}\left|\nabla \tilde{K}^{(r)}\right|_{\sigma \delta_{2}^{14}, \Omega^{t}}+\left|\tilde{K}^{(r)}\right|_{\sigma \delta_{1}^{15}, \Omega^{t}}\left|\nabla v_{1}\right|_{\sigma \delta_{2}^{15}, \Omega^{t}} \\
&+\left|\tilde{K}_{2}\right|_{\sigma \delta_{1}^{16}, \Omega^{t}}\left|\nabla v^{(r)}\right|_{\sigma \delta_{2}^{16}, \Omega^{t}}+\left|H^{(r)}\right|_{\sigma \delta_{1}^{17}, \Omega^{t}}\left|\nabla \tilde{h}_{1}\right|_{\sigma \delta_{2}^{17}, \Omega^{t}} \\
&+\left|H_{2}\right|_{\sigma \delta_{1}^{18}, \Omega^{t}}\left|\nabla \tilde{h}^{(r)}\right|_{\sigma \delta_{2}^{18}, \Omega^{t}}+\left|h^{(r)}\right|_{\sigma \delta_{1}^{19}, \Omega^{t}}\left|\nabla v_{1}\right|_{\sigma \delta_{2}^{19}, \Omega^{t}} \\
&\left.+\left|\tilde{h}_{2}\right|_{\sigma \delta_{1}^{20}, \Omega^{t}}\left|\nabla v^{(r)}\right|_{\sigma \delta_{2}^{20}, \Omega^{t}}\right]
\end{aligned}
$$

for

$$
\frac{1}{\delta_{1}^{k}}+\frac{1}{\delta_{2}^{k}}=1
$$

and $k=1, \ldots, 20$.

It is easy to show that for $20 / 7<\sigma<10 / 3, \eta>4$ we obtain

$$
\begin{aligned}
& \left\|h^{(r)}, K^{(r)}\right\|_{W_{\sigma}^{2,1}\left(\Omega^{t}\right)}+\left|\nabla q^{(r)}\right|_{\sigma, \Omega^{t}} \\
& \quad \leq\left\|A^{(r)}\right\|_{W_{2}^{2,1}\left(\Omega^{t}\right)}\|\tilde{h}, \tilde{K}\|_{\mathcal{M}\left(\Omega^{t}\right)}+\|A\|_{W_{2}^{2,1}\left(\Omega^{t}\right)}\left\|\tilde{h}^{(r)}, \tilde{K}^{(r)}\right\|_{\mathcal{M}\left(\Omega^{t}\right)} .
\end{aligned}
$$


Assume that $\tilde{h}_{s}, \tilde{K}_{s}, s=1,2$ belongs to a bounded set in $\mathcal{M}\left(\Omega^{T}\right)$. Hence, there exists $L$ such that

$$
\left\|\tilde{h}_{s}, \tilde{K}_{s}\right\|_{\mathcal{M}\left(\Omega^{T}\right)} \leq L, \quad\left\|A_{s}\right\|_{W_{2}^{2,1}\left(\Omega^{T}\right)} \leq \varphi(L)
$$

Therefore

$$
\begin{aligned}
\left\|h^{(r)}, K^{(r)}\right\|_{W_{\sigma}^{2,1}\left(\Omega^{t}\right)}+\left\|\nabla q^{(r)}\right\|_{L_{\sigma}\left(\Omega^{t}\right)} \leq & c(L)\left\|A^{(r)}\right\|_{W_{2}^{2,1}\left(\Omega^{t}\right)} \\
& +\phi(L)\left\|\tilde{h}^{(r)}, \tilde{K}^{(r)}\right\|_{\mathcal{M}\left(\Omega^{t}\right) .}
\end{aligned}
$$

Thus, to show continuity of the transformation $\Phi$ we should find an estimate for $\left\|A^{(r)}\right\|_{W_{2}^{2,1}\left(\Omega^{t}\right)}$.

For this purpose we consider the following problems:

$$
\begin{aligned}
& v_{, t}^{(r)}-\operatorname{div} \mathbb{T}\left(v^{(r)}, p^{(r)}\right)=-v^{(r)^{\prime}} \cdot \nabla^{\prime} v_{1}-v_{2} \cdot \nabla^{\prime} v^{(r)} \\
& -w^{(r)} \tilde{h}_{1}-w_{2} \tilde{h}^{(r)}+H^{(r)^{\prime}} \cdot \nabla^{\prime} H_{1}+H_{2}^{\prime} \cdot \nabla^{\prime} H^{(r)} \\
& +\mu^{(r)} \tilde{K}_{1}+\mu_{2} \tilde{K}^{(r)}-H_{i}^{(r)} \cdot \nabla H_{1 i} \\
& -H_{2 i} \cdot \nabla H_{i}^{(r)}-\mu^{(r)} \cdot \nabla \mu_{1}-\mu_{2} \cdot \nabla \mu^{(r)} \quad \text { in } \Omega^{T}, \\
& \operatorname{div} v^{(r)}=0 \\
& \text { in } \Omega^{T} \text {, } \\
& \bar{n} \cdot \mathbb{T}\left(v^{(r)}, p^{(r)}\right) \cdot \bar{\tau}_{\alpha}=v^{(r)} \cdot \bar{n}=0, \quad \alpha=1,2, \quad \text { on } S^{T}, \\
& \left.v^{(r)}\right|_{t=0}=0 \\
& \text { in } \Omega \text {, }
\end{aligned}
$$

and

$$
\begin{array}{ll}
H_{, t}^{(r)}-\nu_{\varkappa} \Delta H^{(r)}=v^{(r)^{\prime}} \cdot \nabla H_{1}+v_{2}^{\prime} \cdot \nabla^{\prime} H^{(r)}-w^{(r)} \tilde{K}_{1} \\
\quad-w_{2} \tilde{K}^{(r)}+H^{(r)^{\prime}} \cdot \nabla^{\prime} v_{1}+H_{2}^{\prime} \cdot \nabla^{\prime} v^{(r)}+\mu^{(r)} \tilde{h}_{1} & \\
\quad+\mu_{2} \tilde{h}^{(r)} & \text { in } \Omega^{T}, \\
\bar{n} \cdot H^{(r)}=0, \quad \bar{n} \cdot \mathbb{D}\left(H^{(r)}\right) \cdot \bar{\tau}_{\alpha}=0, \quad \alpha=1,2, & \text { on } S^{T}, \\
\left.H^{(r)}\right|_{t=0}=0 & \text { in } \Omega,
\end{array}
$$

where $v^{(r)^{\prime}}=\left(v_{1}^{(r)}, v_{2}^{(r)}\right), w^{(r)}=v_{3}^{(r)}, \mu^{(r)}=H_{3}^{(r)}, v_{s}^{\prime}=\left(v_{s 1}, v_{s 2}\right), w_{s}=v_{s 3}$.

For solutions of (4.26) and (4.27) we have

$$
\begin{aligned}
\left\|A^{(r)}\right\|_{W_{2}^{2,1}\left(\Omega^{t}\right)} & +|\nabla p|_{2, \Omega^{t}} \leq\left. c|| v^{(r)^{\prime}} \nabla^{\prime} v_{1}\right|_{2, \Omega^{t}}+\left|v_{2}^{\prime} \nabla^{\prime} v^{(r)}\right|_{2, \Omega^{t}} \\
& +\left|w^{(r)} h_{1}\right|_{2, \Omega^{t}}+\left|w_{2} h^{(r)}\right|_{2, \Omega^{t}}+\left|H^{(r)^{\prime}} \nabla^{\prime} H_{1}\right|_{2, \Omega^{t}} \\
& +\left|H_{2}^{\prime} \nabla^{\prime} H^{(r)}\right|_{2, \Omega^{t}}+\left|\mu^{(r)} K_{1}\right|_{2, \Omega^{t}}+\left|\mu_{2} K^{(r)}\right|_{2, \Omega^{t}} \\
& +\left|H_{i}^{(r)} \nabla H_{1 i}\right|_{2, \Omega^{t}}+\left|H_{2 i} \nabla H_{i}^{(r)}\right|_{2, \Omega^{t}}+\left|\mu^{(r)} \nabla \mu_{1}\right|_{2, \Omega^{t}} \\
& +\left|\mu_{2} \nabla \mu^{(r)}\right|_{2, \Omega^{t}}+\left|v^{(r)^{\prime}} \nabla^{\prime} H_{1}\right|_{2, \Omega^{t}}+\left|v_{2}^{\prime} \nabla^{\prime} H^{(r)}\right|_{2, \Omega^{t}} \\
& +\left|w^{(r)} K_{1}\right|_{2, \Omega^{t}}+\left|w_{2} K^{(r)}\right|_{2, \Omega^{t}}+\left|H^{(r)^{\prime}} \nabla^{\prime} v_{1}\right|_{2, \Omega^{t}} \\
& \left.+\left|H_{2}^{\prime} \nabla^{\prime} v^{(r)}\right|_{2, \Omega^{t}}+\left|\mu^{(r)} h_{1}\right|_{2, \Omega^{t}}+\left|\mu_{2} h^{(r)}\right|_{2, \Omega^{t}}\right] .
\end{aligned}
$$


We estimate the r.h.s. of (4.28). First we have

$$
\left|v^{(r)^{\prime}} \nabla^{\prime} v_{1}\right|_{2, \Omega^{t}} \leq c\left|v^{(r)}\right|_{5, \Omega^{t}}\left\|v_{1}\right\|_{W_{2}^{2,1}\left(\Omega^{t}\right)}=I_{1} .
$$

By interpolation we get

$$
\begin{aligned}
& I_{1} \leq \varepsilon_{1}\left\|v^{(r)}\right\|_{W_{2}^{2,1}\left(\Omega^{t}\right)}+c\left(1 / \varepsilon_{1}\right) \varphi\left(\left\|v_{1}\right\|_{W_{2}^{2,1}\left(\Omega^{t}\right)}\right)\left|v^{(r)}\right|_{2, \Omega^{t}}, \\
& \left|v_{2} \cdot \nabla^{\prime} v^{(r)}\right|_{2, \Omega^{t}} \leq \varepsilon_{2}\left\|v^{(r)}\right\|_{W_{2}^{2,1}\left(\Omega^{t}\right)}+c\left(1 / \varepsilon_{2}\right) \varphi\left(\left\|v_{2}\right\|_{W_{2}^{2,1}\left(\Omega^{t}\right)}\right)\left|v^{(r)}\right|_{2, \Omega^{t}}, \\
& \left|w^{(r)} h_{1}\right|_{2, \Omega^{t}} \leq c\left|w^{(r)}\right|_{\sigma_{1}, \Omega^{t}}\left|h_{1}\right|_{\sigma_{2}, \Omega^{t}} \equiv I_{3},
\end{aligned}
$$

where $5 / 2-5 / \sigma_{1} \leq 2, \sigma_{2} \leq \infty, 1 / \sigma_{1}+1 / \sigma_{2}=1 / 2, \sigma_{1}<10$.

Because $5 / 2-5 / \sigma_{1}<2$, we have

$$
\begin{gathered}
I_{3} \leq \varepsilon_{3}\left\|v^{(r)}\right\|_{W_{2}^{2,1}\left(\Omega^{t}\right)}+c\left(1 / \varepsilon_{3}\right) \varphi\left(\left\|h_{1}\right\|_{\mathcal{M}\left(\Omega^{t}\right)}\right)\left|v^{(r)}\right|_{2, \Omega^{t}}, \\
\left|w_{2} h^{(r)}\right|_{2, \Omega^{t}} \leq c\left|w_{2}\right|_{\varrho_{1}, \Omega^{t}}\left|h^{(r)}\right|_{\varrho_{2}, \Omega^{t}} \equiv I_{4},
\end{gathered}
$$

where $1 / \varrho_{1}+1 / \varrho_{2}=1 / 2,5 / 2-5 / \varrho_{1} \leq 2$ and we can choose $\varrho_{2}=5 / 2$. Hence

$$
\begin{gathered}
I_{4} \leq c\left\|v_{2}\right\|_{W_{2}^{2,1}\left(\Omega^{t}\right)}\left\|h^{(r)}\right\|_{L_{5 / 2}\left(\Omega^{t}\right)}, \\
\left|H^{(r)^{\prime}} \nabla H_{1}^{\prime}\right|_{2, \Omega^{t}}+\left|H_{i}^{(r)} \nabla H_{1 i}\right|_{2, \Omega^{t}} \leq c\left|H^{(r)}\right|_{5, \Omega^{t}}\left\|H_{1}\right\|_{W_{2}^{2,1}\left(\Omega^{t}\right)} \\
+c\left|H_{i}^{(r)}\right|_{2, \Omega^{t}}\left|H_{1 i}\right|_{W_{2}^{2,1}\left(\Omega^{t}\right)} \equiv I_{5},
\end{gathered}
$$

where

$$
\begin{aligned}
& I_{5} \leq \varepsilon_{5}\left\|H^{(r)}\right\|_{W_{2}^{2,1}\left(\Omega^{t}\right)}+c\left(1 / \varepsilon_{5}\right) \varphi\left(\left\|H_{1}\right\|_{W_{2}^{2,1}\left(\Omega^{t}\right)}\right)\left\|H^{(r)}\right\|_{L_{2}\left(\Omega^{t}\right)}, \\
& \left|H_{2}^{\prime} \nabla^{\prime} H^{(r)}\right|_{2, \Omega^{t}} \leq \varepsilon_{6}\left\|H^{(r)}\right\|_{W_{2}^{2,1}\left(\Omega^{t}\right)}+c\left(1 / \varepsilon_{6}\right) \varphi\left(\left\|H_{2}\right\|_{W_{2}^{2,1}\left(\Omega^{t}\right)}\right)\left|H^{(r)}\right|_{2, \Omega^{t}}, \\
& \left|\mu^{(r)} K_{1}\right|_{2, \Omega^{t}} \leq \varepsilon_{7}\left\|H^{(r)}\right\|_{W_{2}^{2,1}\left(\Omega^{t}\right)}+c\left(1 / \varepsilon_{7}\right) \varphi\left(\left\|K_{1}\right\|_{\mathcal{M}\left(\Omega^{t}\right)}\right)\left|H^{(r)}\right|_{2, \Omega^{t}}, \\
& \left|\mu_{2} K^{(r)}\right|_{2, \Omega^{t}} \leq c\left\|H_{2}\right\|_{W_{2}^{2,1}\left(\Omega^{t}\right)}\left|K^{(r)}\right|_{5 / 2, \Omega^{t}}, \\
& \left|H_{2 i} \nabla H_{i}^{(r)}\right|_{2, \Omega^{t}} \leq \varepsilon_{8}\left\|H^{(r)}\right\|_{W_{2}^{2,1}\left(\Omega^{t}\right)}+c\left(1 / \varepsilon_{8}\right) \varphi\left(\left\|H_{2}\right\|_{W_{2}^{2,1}\left(\Omega^{t}\right)}\right)\left|H^{(r)}\right|_{2, \Omega^{t}}, \\
& \left|\mu^{(r)} \nabla \mu_{1}\right|_{2, \Omega^{t}}=\left|H_{3}^{(r)} \nabla H_{13}\right|_{2, \Omega^{t}} \\
& \quad \leq \varepsilon_{9}\left\|H^{(r)}\right\|_{W_{2}^{2,1}\left(\Omega^{t}\right)}+c\left(1 / \varepsilon_{9}\right) \varphi\left(\left\|H_{1}\right\|_{W_{2}^{2,1}\left(\Omega^{t}\right)}\right)\left|H^{(r)}\right|_{2, \Omega^{t}}, \\
& \left|\mu_{2} \nabla \mu^{(r)}\right|_{2, \Omega^{t}} \leq \varepsilon_{10}\left\|H^{(r)}\right\|_{W_{2}^{2,1}\left(\Omega^{t}\right)}+c\left(1 / \varepsilon_{10}\right) \varphi\left(\left\|H_{2}\right\|_{W_{2}^{2,1}\left(\Omega^{t}\right)}\right)\left|H^{(r)}\right|_{2, \Omega^{t}}, \\
& \left|v^{(r)^{\prime}} \nabla^{\prime} H_{1}\right|_{2, \Omega^{t}}+\left|v_{2}^{\prime} \nabla^{\prime} H^{(r)}\right|_{2, \Omega^{t}}+\left|H^{(r)^{\prime}} \nabla^{\prime} v_{1}\right|_{2, \Omega^{t}}+\left|H_{2}^{\prime} \nabla^{\prime} v^{(r)}\right|_{2, \Omega^{t}} \\
& \leq \varepsilon_{11}\left\|v^{(r)}, H^{(r)}\right\|_{W_{2}^{2,1}\left(\Omega^{t}\right)}+c\left(1 / \varepsilon_{11}\right) \varphi\left(\left\|H^{\prime}\right\|_{W_{2}^{2,1}\left(\Omega^{t}\right)},\left\|v^{\prime}\right\|_{W_{2}^{2,1}\left(\Omega^{t}\right)}\right) H^{(r)},\left.v^{(r)}\right|_{2, \Omega^{t},}, \\
& \left|w^{(r)} K_{1}\right|_{2, \Omega^{t}}+\left|w_{2} K^{(r)}\right|_{2, \Omega^{t}} \leq \varepsilon_{12}\left\|v^{(r)}\right\|_{W_{2}^{2,1}\left(\Omega^{t}\right)} \\
& \quad+c\left(1 / \varepsilon_{12}\right) \varphi\left(\left\|K_{1}\right\|_{\mathcal{M}\left(\Omega^{t}\right)}\right)\left|v^{(r)}\right|_{2, \Omega^{t}}+c\left\|v_{2}\right\|_{W_{2}^{2,1}\left(\Omega^{t}\right)}\left|K^{(r)}\right|_{5 / 2, \Omega^{t},},
\end{aligned}
$$




$$
\begin{gathered}
\left|H^{(r)^{\prime}} \nabla^{\prime} v_{1}\right|_{2, \Omega^{t}}+\left|H_{2}^{\prime} \nabla^{\prime} v^{(r)}\right|_{2, \Omega^{t}} \leq \varepsilon_{13}\left\|H^{(r)}\right\|_{W_{2}^{2,1}\left(\Omega^{t}\right)} \\
+c\left(1 / \varepsilon_{13}\right) \varphi\left(\left\|v_{1}\right\|_{W_{2}^{2,1}\left(\Omega^{t}\right)}\right)\left|H^{(r)}\right|_{2, \Omega^{t}}+\varepsilon_{14}\left\|v^{(r)}\right\|_{W_{2}^{2,1}\left(\Omega^{t}\right)} \\
+c\left(1 / \varepsilon_{14}\right) \varphi\left(\left\|H_{2}\right\|_{W_{2}^{2,1}\left(\Omega^{t}\right)}\right)\left|v^{(r)}\right|_{2, \Omega^{t}}, \\
\left|\mu^{(r)} h_{1}\right|_{2, \Omega^{t}}+\left|\mu_{2} h^{(r)}\right|_{2, \Omega^{t}} \leq \varepsilon_{15}\left\|H^{(r)}\right\|_{W_{2}^{2,1}\left(\Omega^{t}\right)} \\
+c\left(1 / \varepsilon_{15}\right) \varphi\left(\left\|h_{1}\right\|_{\mathcal{M}\left(\Omega^{t}\right)}\right)\left|H^{(r)}\right|_{2, \Omega^{t}}+c\left\|H_{2}\right\|_{W_{2}^{2,1}\left(\Omega^{t}\right)}\left|h^{(r)}\right|_{5 / 2, \Omega^{t}} .
\end{gathered}
$$

Utilizing the above estimates in (4.28) and assuming that $\varepsilon_{1}, \ldots, \varepsilon_{15}$ are sufficiently small we obtain

$$
\begin{aligned}
\left\|A^{(r)}\right\|_{W_{2}^{2,1}\left(\mid \Omega^{t}\right)}+\left|\nabla p^{(r)}\right|_{2, \Omega^{t}} & \\
\leq & \varphi\left(\left\|v_{1}, v_{2}\right\|_{W_{2}^{2,1}\left(\Omega^{t}\right)},\left\|H_{1}, H_{2}\right\|_{W_{2}^{2,1}\left(\Omega^{t}\right)},\left\|h_{1}, K_{1}\right\|_{W_{\sigma}^{2,1}\left(\Omega^{t}\right)}\right) \\
& \cdot\left(\left\|A^{(r)}\right\|_{L_{2}\left(\Omega^{t}\right)}+\left|K^{(r)}, h^{(r)}\right|_{5 / 2, \Omega^{t}}\right) .
\end{aligned}
$$

Hence

$$
\left\|A^{(r)}\right\|_{W_{2}^{2,1}\left(\Omega^{t}\right)}+\left|\nabla p^{(r)}\right|_{2, \Omega^{t}} \leq \varphi(L)\left(\left|A^{(r)}\right|_{2, \Omega^{t}}+\left|K^{(r)}, h^{(r)}\right|_{5 / 2, \Omega^{t}}\right) .
$$

Finally we estimate the r.h.s. of (4.30).

We start with energy estimates on $h^{(r)}$ and $K^{(r)}$.

Multiplying $(4.19)_{1}$ by $h^{(r)},(4.20)_{1}$ by $K^{(r)}$ and integrating over $\Omega$ yields

$$
\frac{1}{2} \frac{d}{d t}\left|h^{(r)}, K^{(r)}\right|_{2, \Omega}^{2}+\bar{\nu}\left|\nabla h^{(r)}, \nabla K^{(r)}\right|_{2, \Omega}^{2}=\int_{\Omega} \psi_{1} h^{(r)} d x+\int_{\Omega} \psi_{2} K^{(r)} d x
$$

where $\psi_{1}$ and $\psi_{2}$ are the r.h.s. of $(4.19)_{1},(4.20)_{1}$, respectively. By the Hölder inequality, we have

$$
\frac{d}{d t}\left|h^{(r)}, K^{(r)}\right|_{2, \Omega}^{2}+\bar{\nu}\left\|h^{(r)}, K^{(r)}\right\|_{H^{1}(\Omega)}^{2} \leq c\left[\left|v^{(r)}\right|_{2, \Omega}^{2}\left|\nabla \tilde{h}_{1}\right|_{3, \Omega}^{2}\right.
$$

$$
\begin{aligned}
& +\left|v_{2}\right|_{6, \Omega}^{2}\left|\tilde{h}^{(r)}\right|_{3, \Omega}^{2}+\left|\tilde{h}_{2}\right|_{3, \Omega}^{2}\left|\nabla v^{(r)}\right|_{2, \Omega}^{2}+\left|\tilde{h}^{(r)}\right|_{2, \Omega}^{2}\left|\nabla v_{1}\right|_{3, \Omega}^{2}+\left|\tilde{K}^{(r)}\right|_{3, \Omega}^{2}\left|\nabla H_{1}\right|_{2, \Omega}^{2} \\
& +\left|\tilde{K}_{2}\right|_{3, \Omega}^{2}\left|\nabla H^{(r)}\right|_{2, \Omega}^{2}+\left|H^{(r)}\right|_{2, \Omega}^{2}\left|\nabla \tilde{K}_{1}\right|_{3, \Omega}^{2}+\left|\nabla H_{2}\right|_{2, \Omega}^{2}\left|\tilde{K}^{(r)}\right|_{3, \Omega}^{2} \\
& +\left|\tilde{h}^{(r)}\right|_{3, \Omega}^{2}\left|H_{1}\right|_{6, \Omega}^{2}+\left|\tilde{h}_{2}\right|_{3, \Omega}^{2}\left|\nabla H^{(r)}\right|_{2, \Omega}^{2}+\left|v^{(r)}\right|_{2, \Omega}^{2}\left|\nabla \tilde{K}_{1}\right|_{3, \Omega}^{2} \\
& \left.+\left|v_{2}\right|_{6, \Omega}^{2}\left|\tilde{K}^{(r)}\right|_{3, \Omega}^{2}+\left|H^{(r)}\right|_{2, \Omega}^{2}\left|\nabla \tilde{h}_{1}\right|_{3, \Omega}^{2}+\left|H_{2}\right|_{6, \Omega}^{2}\left|\tilde{h}^{(r)}\right|_{3, \Omega}^{2}\right] .
\end{aligned}
$$

Using (4.24) in (4.32) we obtain

$$
\begin{aligned}
& \frac{d}{d t}\left|h^{(r)}, K^{(r)}\right|_{2, \Omega}^{2}+\bar{\nu}\left\|h^{(r)}, K^{(r)}\right\|_{H^{1}(\Omega)}^{2} \leq c\left(\varphi ( L ) \left(\left\|v^{(r)}\right\|_{H^{1}(\Omega)}^{2}\right.\right. \\
& \left.\quad+\left\|H^{(r)}\right\|_{H^{1}(\Omega)}^{2}\right)+\left|v_{2}\right|_{6, \Omega}^{2}\left|\tilde{h}^{(r)}, \tilde{K}^{(r)}\right|_{3, \Omega}^{2} \\
& \left.\quad+\left|v^{(r)}, H^{(r)}\right|_{2, \Omega}^{2}\left|\nabla \tilde{h}_{1}, \nabla \tilde{K}_{1}\right|_{3, \Omega}^{2}+\left|h^{(r)}, K^{(r)}\right|_{2, \Omega}^{2}\left|\nabla v_{1}, \nabla H_{1}\right|_{3, \Omega}^{2}\right) .
\end{aligned}
$$


Multiplying (4.26) $)_{1}$ by $v^{(r)}$ and integrating over $\Omega$, it follows that

$$
\frac{d}{d t}\left|v^{(r)}\right|_{2, \Omega}^{2}+\nu\left\|v^{(r)}\right\|_{H^{1}(\Omega)}^{2}=-\int_{\Omega} \psi_{3} v^{(r)} d x,
$$

where $\psi_{3}$ is the r.h.s. of $(4.26)_{1}$. Estimating the r.h.s. of (4.34) step by step we obtain (with all integrals over $\Omega$ )

$$
\begin{aligned}
& -\int v^{(r)^{\prime}} \nabla v_{1} v^{(r)} d x \leq \varepsilon_{1}\left|v^{(r)}\right|_{6, \Omega}^{2}+c\left(1 / \varepsilon_{1}\right)\left|v^{(r)}\right|_{2, \Omega}^{2}\left|\nabla v_{1}\right|_{3, \Omega}^{2}, \\
& -\int v_{2}^{\prime} \cdot \nabla v^{(r)} v^{(r)} d x=0, \\
& -\int v^{(r)} h_{1} v^{(r)} d x \leq \varepsilon_{2}\left|v^{(r)}\right|_{6, \Omega}^{2}+c\left(1 / \varepsilon_{2}\right)\left|v^{(r)}\right|_{2, \Omega}^{2}\left|h_{1}\right|_{3, \Omega}^{2}, \\
& -\int w_{2} h^{(r)} v^{(r)} d x \leq \varepsilon_{3}\left|v^{(r)}\right|_{6, \Omega}^{2}+c\left(1 / \varepsilon_{3}\right)\left|w_{2}\right|_{3, \Omega}^{2}\left|h^{(r)}\right|_{2, \Omega}^{2}, \\
& \int\left(H^{(r)^{\prime}} \nabla H_{1}+H_{2}^{\prime} \nabla H^{(r)}\right) v^{(r)} d x \leq \varepsilon_{4}\left|v^{(r)}\right|_{6, \Omega}^{2} \\
& +c\left(1 / \varepsilon_{4}\right)\left|H^{(r)}\right|_{2, \Omega}^{2}\left|\nabla H_{1}\right|_{3, \Omega}^{2}+\varepsilon_{5}\left|\nabla H^{(r)}\right|_{2, \Omega}^{2}+c\left(1 / \varepsilon_{5}\right)\left|v^{(r)}\right|_{2, \Omega}^{2}\left|H_{2}\right|_{\infty, \Omega}^{2}, \\
(4.35) \quad & \int \mu^{(r)} K_{1} v^{(r)} d x \leq \varepsilon_{6}\left|v^{(r)}\right|_{6, \Omega}^{2} \\
& \quad+c\left(1 / \varepsilon_{6}\right)\left|\mu^{(r)} K_{1}\right|_{6 / 5, \Omega}^{2} \leq \varepsilon_{6}\left|v^{(r)}\right|_{6, \Omega}^{2}+c(1 / \varepsilon)\left|H^{(r)}\right|_{2, \Omega}\left|K_{1}\right|_{3, \Omega}^{2}, \\
& \int \mu_{2} K^{(r)} v^{(r)} d x \leq \varepsilon_{7}\left|v^{(r)}\right|_{6, \Omega}^{2}+c\left(1 / \varepsilon_{7}\right)\left|\mu_{2}\right|_{3, \Omega}^{2}\left|K^{(r)}\right|_{2, \Omega}^{2}, \\
& -\int\left(H_{i}^{(r)} \nabla H_{1 i}+H_{2 i} \nabla H_{i}^{(r)}\right) v^{(r)} d x \leq \varepsilon_{8}\left|v^{(r)}\right|_{6, \Omega}^{2}+\varepsilon_{9}\left|\nabla H^{(r)}\right|_{2, \Omega}^{2} \\
& +c\left(1 / \varepsilon_{8}\right)\left|H^{(r)}\right|_{2, \Omega}^{2}\left|\nabla H_{1}\right|_{3, \Omega}^{2}+c\left(1 / \varepsilon_{9}\right)\left|v^{(r)}\right|_{2, \Omega}^{2}\left|H_{2}\right|_{\infty, \Omega}^{2}, \\
& -\int \mu^{(r)} \nabla \mu_{1} v^{(r)} d x \leq \varepsilon_{10}\left|v^{(r)}\right|_{6, \Omega}^{2}+c\left(1 / \varepsilon_{10}\right)\left|H^{(r)}\right|_{2, \Omega}^{2}\left|\nabla H_{1}\right|_{3, \Omega}^{2}, \\
& -\int \mu_{2} \nabla \mu^{(r)} v^{(r)} d x \leq \varepsilon_{11}\left|\nabla H^{(r)}\right|_{2, \Omega}^{2}+c\left(1 / \varepsilon_{11}\left|v^{(r)}\right|_{2, \Omega}^{2}\left|H_{2}\right|_{\infty, \Omega}^{2},\right.
\end{aligned}
$$

Next multiplying (4.27) 1 by $H^{(r)}$ and integrating over $\Omega$ we get the estimates

$$
\begin{aligned}
& \int v^{(r)^{\prime}} \nabla H_{1} H^{(r)} d x \leq \varepsilon_{12}\left|\nabla H^{(r)}\right|_{2, \Omega}^{2}+c\left(1 / \varepsilon_{12}\right)\left|v^{(r)}\right|_{2, \Omega}^{2}\left|H_{1}\right|_{\infty, \Omega}^{2}, \\
& \int v_{2}^{\prime} \nabla H^{(r)} H^{(r)} d x=0, \\
& -\int w^{(r)} K_{1} H^{(r)} d x \leq \varepsilon_{13}\left|H^{(r)}\right|_{6, \Omega}^{2}+c\left(1 / \varepsilon_{13}\right)\left|K_{1}\right|_{3, \Omega}^{2}\left|v^{(r)}\right|_{2, \Omega}^{2}, \\
& -\int w_{2} K^{(r)} H^{(r)} d x \leq \varepsilon_{14}\left|H^{(r)}\right|_{6, \Omega}^{2}+c\left(1 / \varepsilon_{14}\right)\left|v_{2}\right|_{3, \Omega}^{2}\left|K^{(r)}\right|_{2, \Omega}^{2}, \\
& \int H^{(r)^{\prime}} \nabla v_{1} H^{(r)} d x \leq \varepsilon_{15}\left|H^{(r)}\right|_{6, \Omega}^{2}+c\left(1 / \varepsilon_{15}\right)\left|\nabla v_{1}\right|_{3, \Omega}^{2}\left|H^{(r)}\right|_{2, \Omega}^{2}, \\
& \int H_{2}^{\prime} \nabla v^{(r)} H^{(r)} d x \leq \varepsilon_{16}\left|\nabla v^{(r)}\right|_{2, \Omega}^{2}+c\left(1 / \varepsilon_{16}\right)\left|H_{2}\right|_{\infty, \Omega}^{2}\left|H^{(r)}\right|_{2, \Omega}^{2}, \\
& \int \mu^{(r)} h_{1} H^{(r)} d x \leq \varepsilon_{17}\left|H^{(r)}\right|_{6, \Omega}^{2}+c\left(1 / \varepsilon_{17}\right)\left|h_{1}\right|_{3, \Omega}^{2}\left|H^{(r)}\right|_{2, \Omega}^{2}, \\
& \int \mu_{2} h^{(r)} H^{(r)} d x \leq \varepsilon_{18}\left|H^{(r)}\right|_{6, \Omega}^{2}+c\left(1 / \varepsilon_{18}\right)\left|\mu_{2}\right|_{3, \Omega}^{2}\left|h^{(r)}\right|_{2, \Omega}^{2} .
\end{aligned}
$$


Taking $\varepsilon_{1}, \ldots, \varepsilon_{18}$ sufficiently small and adding all estimates (4.35)-(4.36) we get

$$
\begin{aligned}
\frac{d}{d t}\left|A^{(r)}\right|_{2, \Omega}^{2}+\nu\left\|A^{(r)}\right\|_{H^{1}(\Omega)}^{2} & \\
\leq & c\left|A^{(r)}\right|_{2, \Omega}^{2}\left[\left|\nabla v_{1}, \nabla H_{1}\right|_{3, \Omega}^{2}+\left|h_{1}, K_{1}\right|_{3, \Omega}^{2}+\left|H_{1}, H_{2}\right|_{\infty, \Omega}^{2}\right] \\
& \quad+c\left|w_{2}, \mu_{2}\right|_{3, \Omega}^{2}\left|h^{(r)}\right|_{2, \Omega}^{2}+c\left|\mu_{2}, v_{2}\right|_{3, \Omega}^{2}\left|K^{(r)}\right|_{2, \Omega}^{2} .
\end{aligned}
$$

Multiplying (4.37) by a constant $c_{*}$ such that $\nu c_{*}-c \phi(L) \geq \nu$ and adding to (4.33), we get

$$
\begin{aligned}
\frac{d}{d t}\left(c_{*}\left|A^{(r)}\right|_{2, \Omega}^{2}+\right. & \left.\left|h^{(r)}, K^{(r)}\right|_{2, \Omega}^{2}\right)+\nu\left(\left\|A^{(r)}\right\|_{H^{1}(\Omega)}^{2}+\left\|h^{(r)}, K^{(r)}\right\|_{H^{1}(\Omega)}^{2}\right) \\
\leq & c_{*} c\left|A^{(r)}\right|_{2, \Omega}^{2}\left[\left|\nabla \tilde{h}_{1}, \nabla \tilde{K}_{1}\right|_{3, \Omega}^{2}+\left|h_{1}, K_{1}\right|_{3, \Omega}^{2}+\left|\nabla v_{1}, \nabla H_{1}\right|_{3, \Omega}^{2}\right. \\
& \left.+\left|H_{1}, H_{2}\right|_{\infty, \Omega}^{2}\right]+c_{*} c\left|v_{2}\right|_{6, \Omega}^{2}\left|\tilde{h}^{(r)}, \tilde{K}^{(r)}\right|_{3, \Omega}^{2} \\
& +c_{*} c\left|h^{(r)}, K^{(r)}\right|_{2, \Omega}^{2}\left(\left|\nabla A_{1}, v_{2}\right|_{3, \Omega}^{2}+\left|w_{2}, \mu_{2}\right|_{3, \Omega}^{2}\right) .
\end{aligned}
$$

Integrating this inequality with respect to time yields

$$
\begin{aligned}
& \left|A^{(r)}\right|_{2, \Omega}^{2}+\left|h^{(r)}, K^{(r)}\right|_{2, \Omega}^{2}+\nu \int_{0}^{t}\left(\left\|A^{(r)}\right\|_{H^{1}(\Omega)}^{2}+\left\|H^{(r)}, K^{(r)}\right\|_{H^{1}(\Omega)}^{2}\right) d \tau \\
& \leq c \exp \left\{\int_{0}^{t}\left|\nabla \tilde{h}_{1}, \nabla \tilde{K}_{1}\right|_{3, \Omega}^{2} d \tau+\int_{0}^{t}\left|h_{1}, K_{1}\right|_{3, \Omega}^{2} d \tau+\int_{0}^{t}\left|\nabla v_{1}, \nabla H_{1}\right|_{3, \Omega}^{2} d \tau\right. \\
& \left.\quad+\int_{0}^{t}\left|H_{1}, H_{2}\right|_{\infty, \Omega}^{2} d \tau+\int_{0}^{t}\left|\nabla A_{1}, v_{2}\right|_{3, \Omega}^{2} d \tau+\int_{0}^{t}\left|w_{2}, \mu_{2}\right|_{3, \Omega}^{2} d \tau\right\} \\
& \quad \cdot\left(\int_{0}^{t}\left|v_{2}\right|_{6, \Omega}^{2}\left|\tilde{h}^{(r)}, \tilde{K}^{(r)}\right|_{3, \Omega}^{2} d \tau\right. \\
& \left.\quad+\left\|\nabla A_{1}\right\|_{L_{2}\left(0, t ; L_{3}(\Omega)\right)}^{2}\left|\tilde{h}^{(r)}, \tilde{K}^{(r)}\right|_{L_{\infty}\left(0, t ; L_{2}(\Omega)\right)}\right) \equiv J .
\end{aligned}
$$

By suitable imbedding results we get

$$
\begin{aligned}
J \leq & c \exp \left\{\left\|A_{1}\right\|_{W_{2}^{2,1}\left(\Omega^{t}\right)}^{2}+\|h, K\|_{W_{\sigma}^{2,1}\left(\Omega^{t}\right)}^{2}\right\} \\
& \cdot\left(\sup _{t}\left|\tilde{h}^{(r)}, \tilde{K}^{(r)}\right|_{3, \Omega}^{2}\left\|v_{2}\right\|_{H^{1}(\Omega)}^{2}+\sup _{t}\left|\tilde{h}^{(r)}, \tilde{K}^{(r)}\right|_{3, \Omega}^{2} \int_{0}^{t}\left|\nabla A_{1}\right|_{3, \Omega}^{2} d z\right) \\
\equiv & J_{1} .
\end{aligned}
$$

By (4.14), (4.18) and (4.24) we obtain

$$
J_{1} \leq \varphi(L)\left[\left\|\tilde{h}^{(r)}, \tilde{K}^{(r)}\right\|_{L_{\infty}\left(0, t ; L_{3}(\Omega)\right)}^{2}+\left\|\tilde{h}^{(r)}, \tilde{K}^{(r)}\right\|_{L_{\infty}\left(0, t ; L_{2}(\Omega)\right.}^{2}\right] .
$$


Therefore, (4.38) takes the form

$$
\begin{aligned}
&\left\|A^{(r)}\right\|_{V_{2}^{0}\left(\Omega^{t}\right)}+\left\|h^{(r)}, K^{(r)}\right\|_{V_{2}^{0}\left(\Omega^{t}\right)} \\
& \leq \varphi(L)\left\|\tilde{h}^{(r)}, \tilde{K}^{(r)}\right\|_{L_{\infty}\left(0, t ; L_{3}(\Omega)\right.} .
\end{aligned}
$$

Utilizing (4.39) in (4.30) and the result in (4.25) we obtain

$$
\left\|h^{(r)}, K^{(r)}\right\|_{\mathcal{M}\left(\Omega^{T}\right)} \leq \varphi(L)\left\|\tilde{h}^{(r)}, \tilde{K}^{(r)}\right\|_{\mathcal{M}\left(\Omega^{T}\right)},
$$

which implies the uniform continuity of $\Phi$ and ends the proof.

Proof of Theorem 1. Since $\Phi$ is uniformly continuous and compact for $20 / 7<\sigma \leq 10 / 3$, the Leray-Schauder fixed point theorem yields the existence result.

Moreover, for $5 / 3<\sigma<3$, by (3.28), Lemma 3.5 and Lemma 3.3 we have estimates of the form (1.3). This concludes the proof.

Acknowledgments. The second author is partially supported by Polish Grant No. NN 201396937.

\section{References}

[1] O. V. Besov, V. P. Il'in and S. N. Nikol'skiǔ, Integral Representation of Functions and Imbedding Theorems, Nauka, Moscow, 1975 (in Russian).

[2] P. Kacprzyk, Almost global solutions of the free boundary problem for the equations of a magnetohydrodynamic incompressible fluid, Appl. Math. (Warsaw) 31 (2004), 69-77.

[3] - Global existence of solutions of the free boundary problem for the equations of magnetohydrodynamic incompressible viscous fluid, Topol. Methods Nonlinear Anal. 23 (2004), 339-356.

[4] - Free boundary problem for the equations of magnetohydrodynamic incompressible viscous fluid, Appl. Math. (Warsaw) 34 (2007), 75-95.

[5] O. A. Ladyzhenskaya, Mathematical Theory of Viscous Incompressible Flow, Nauka, Moscow, 1970 (in Russian).

[6] L. Landau and E. Lifshits, Electrodynamics of Continuous Media, Gos. Izdat. Tekhn.Teor. Lit., Moscow, 1957 (in Russian).

[7] B. Nowakowski and W. M. Zajączkowski, Very weak solutions to the boundary value problem of the homogeneous heat equation, to appear.

[8] J. Rencławowicz and W. M. Zajacczkowski, Large time regular solutions to the Navier-Stokes equations in cylindrical domains, Topol. Methods Nonlinear Anal. 32 (2008), 69-87.

[9] V. A. Solonnikov and V. E. Schadilov, On a boundary value problem for a stationary system of the Navier-Stokes equations, Trudy Mat. Inst. Steklova 125 (1973), 196210 (in Russian); English transl.: Proc. Steklov Inst. Math. 125 (1973), 186-199.

[10] W. M. Zajączkowski, Global existence of axially symmetric solutions to the NavierStokes equations with large angular component of velocity, Colloq. Math. 100 (2004), 243-263.

[11] - Long time existence of regular solutions to the Navier-Stokes equations in cylindrical domains under boundary slip conditions, Studia Math. 169 (2005), 243-285. 
[12] W. M. Zajączkowski, Global special regular solutions to the Navier-Stokes equations in a cylindrical domain without the axis of symmetry, Topol. Methods Nonlinear Anal. 24 (2004), 69-105.

[13] - On global regular solutions to the Navier-Stokes equations in cylindrical domains, ibid., to appear.

Wisam Alame

Faculty of Mathematics and Information Sciences

Warsaw University of Technology

Pl. Politechniki 1

00-661 Warszawa, Poland
Wojciech M. Zajaczkowski Institute of Mathematics Polish Academy of Sciences Śniadeckich 8 00-956 Warszawa, Poland E-mail: wz@impan.pl and

Institute of Mathematics and Cryptology Cybernetics Faculty Military University of Technology

Kaliskiego 2 00-908 Warszawa, Poland

Received on 30.1.2008;

revised version on 26.1.2011 